\title{
Finite element method for incompressible viscous flow with immersed pressure jumps with applications to actuator disks and microfluidics
}

\author{
Roberto AUSAS ${ }^{1}$ Gustavo BUSCAGLIA ${ }^{a}$ Vitoriano RUAS b,c \\ a Universidade de São Paulo, Instituto de Ciências Matemáticas e de Computação, São Carlos, BRAZIL \\ b Sorbonne Universit, UMR 7190, CNRS, Institut Jean Le Rond dAlembert, F-75005, Paris, FRANCE \\ ${ }^{\mathrm{c}} \mathrm{CNPq}$ Research grant holder, PUC-Rio, Dept. of Mechanical Engineering, Rio de Janeiro, BRAZIL
}

\begin{abstract}
We propose a finite element method for the solution of viscous incompressible flow problems with singular forces at immersed interfaces. The method combines the Algebraic Subgrid Scale method with a pressure jump stabilization. It consists of the addition, to the continuity equation, of a term weighting the residual of the pressure jump. This term enhances the stability irrespective of possible badly-shaped intersections of the interface with the finite elements. We assess the new method by comparing with the unstabilized case showing improved accuracy and robustness. The examples consider immersed actuator disk problems and one application to thermocapillary convection.
\end{abstract}

Key words: Finite elements, Incompressible viscous flows, Navier-Stokes, Pressure jump, Immersed boundary, Surface tension.

\section{Introduction}

Simulation of fluid flow in several industrial problems requires taking into consideration singular forces at internal interfaces. An important example is the simulation of wind turbines, ship propellers and cooling fans by means of the actuator disk model (see e.g. $[1,2,3]$ ). This model replaces the individual action of the blades of the turbine (or propeller, or fan) by a singular force distributed on an immersed imaginary

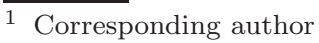

Email addresses: rfausas@icmc.usp.br (Roberto AUSAS), gustavo.buscaglia@icmc.usp.br (Gustavo BUSCAGLIA), vitoriano.ruas@upmc.fr (Vitoriano RUAS).
} 
disk that represents the whole rotor. Singular forces also arise, for example, in the simulation of surface tension phenomena in multiphase flows.

Actuator disk models, or immersed singular forces in general, are especially useful when combined with non-conforming meshes; i.e., meshes that do not conform to the geometrical location of the interfaces at which the forces arise. This allows one to use a single mesh to explore different geometrical configurations, thus reducing the pre-processing burden that can dominate the CFD design pipeline. Non-conforming meshes, however, require very robust approximation methods, that do not loose stability when the intersection of the interface with the grid cells happens to be oddly shaped. Indeed the accurate approximation of pressure and velocity exhibiting strong and weak discontinuities at immersed interfaces remains numerically challenging and has attracted intensive research in the last years. In the finite element literature modifications or enrichment of standard finite element spaces have been proposed to better accommodate the discontinuities and to obtain improved convergence rates. Among these, the XFEM method introduced in [26] is well known (see also [7,8] for applications to two phase flow problems). Elementwise condensable enrichment can be found in the literature $[28,24,9]$ or locally modified spaces [11] which do not alter the structure of the global system of equations to be solved. Variants of Nitsche's methods were also proposed to weakly enforce coupling conditions at immersed interfaces. Together with special function spaces, stabilization techniques are essential for robustness and accuracy. In $[4,5,6]$ significant efforts have been devoted to tackle such issues. There, stabilization methods are proposed to attain improved accuracy and to avoid ill-conditioning of the linear systems resulting from the so called bad-cut elements. All these developments have mainly focused on multiphase flow models, and have not been assessed or optimized for general interfaces with singular forces, such as those that appear in actuator disk models. It is noticeable that our method avoids remeshing in order to conform to a moving interface where singular forces act, like in other known approaches that can be used to tackle such a problem.

In [13], a stabilization of the pressure jump across an interface as a result of prescribed singular forces has been proposed. The method is close to the one studied in [12]. Both are based on a Petrov-Galerkin formulation of the Stokes problem of the type proposed by Franca, Hughes and Balestra [20] or Douglas and Wang [17], depending on a mesh-dependent parameter $\delta$. The main difference between them is the addition, in [13], of a pressure jump term multiplied by another mesh-dependent parameter $\varepsilon>0$. Such a term penalizes errors in the mechanical equilibrium at the immersed interface, increasing the accuracy of the imposition of the singular force distribution. In this article we explore this term for problems with singular forces such as those arising from the actuator disk model. We combine this extra penalty with the Algebraic Subgrid Scale (ASGS) method of Codina [22]. The ASGS variant is a popular generalization of the methods in $[20,17]$ that includes transient and inertial effects. The combined method is an ASGS method itself, now incorporating the aforementioned pressure jump term which is very easy to implement. As shown by numerical experiments, the proposed method is insensitive to bad-cut elements and yields solutions with enhanced accuracy at the interface. A specific formula is proposed for $\varepsilon$ after extensive tests, leaving a method with no free parameters.

An outline of the paper is as follows. In Section 2 we formalize the mathematical setting, while in Section 3 we recall the finite element method of [13] and its combination with the ASGS method for the Navier-Stokes equations. We also give a priori criteria for the choice of method's parameters $\delta$ and $\varepsilon$. In section 4 we perform a series of numerical experiments considering both Stokes and Navier-Stokes regimes, with examples involving immersed actuator disks and a microfluidic application related to the thermocapillary Marangoni migration of a droplet. We conclude in Section 5 with a few remarks on the whole work. 


\section{Mathematical setting}

We consider the flow of a viscous incompressible fluid with dynamic viscosity $\mu$ and density $\rho$, occupying two complementary sub-domains $\Omega_{1}$ and $\Omega_{2}$ of a bounded domain $\Omega \in \mathbb{R}^{2}$ with boundary $\Gamma$, separated by an interface $\Sigma$. Although in this article we restrict ourselves to the two dimensional case for simplicity, the extension to three dimensions is straighforward. Akin to [12], in this method basically a standard continuous piecewise linear representation of both velocity and pressure is adopted. However, in order to take into account discontinuities across $\Sigma$, the pressure interpolation is modified in the elements cut by it in the way specified in [11] that we recall hereafter. In [12] and also in [13] when $N=2, \Sigma$ was assumed to be a Dini-smooth open arc (see e.g. [21]) intersecting $\Gamma$ at exactly two distinct points or a closed Dini-smooth Jordan curve [21] completely immersed in $\Omega$, or yet polygonal counterparts of both situations. Although we keep these assumptions here for the purpose of the formal results given in Section 3, they are not really fulfilled in all the numerical experiments performed in this work. On the other hand, akin to both papers, $\Omega$ is still assumed to be either a polygon or a domain such that $\Gamma$ is of the piecewise $C^{1}$ class. For the same purpose we also assume that a non empty intersection of $\Sigma$ and $\Gamma$ does not contain a vertex of $\Gamma$ in case $\Omega$ is a polygon, and the sinus $\sigma$ of any angle between $\Sigma$ and $\Gamma$ is not zero.

The problem to solve can be stated as follows:

Let $\mathbf{f}$ be a given force field in $\left[L^{2}(\Omega)\right]^{2}$, and $\varphi$ be a given force distribution on $\Sigma$ belonging to $C^{0}(\Sigma)$. Prescribing a velocity $\mathbf{g} \in\left[C^{0}(\Gamma)\right]^{2}$ satisfying $\int_{\Gamma} \mathbf{g} \cdot \mathbf{n}=0$, where $\mathbf{n}$ is the unit outer normal vector on $\Gamma$, we wish to find a velocity $\mathbf{u} \in\left[H^{1}(\Omega)\right]^{2}$ and a pressure $p \in H^{1}(\Omega \backslash \Sigma) \cap L_{0}^{2}(\Omega)$ satisfying the following Navier-Stokes system:

$$
\begin{cases}\rho\left(\partial_{t} \mathbf{u}+\mathbf{u} \cdot \nabla \mathbf{u}\right)-\mu \Delta \mathbf{u}+\nabla p=\mathbf{f} & \text { in } \Omega \backslash \Sigma \\ \operatorname{div} \mathbf{u}=0 & \text { in } \Omega \\ \llbracket p \rrbracket=\varphi & \text { on } \Sigma \\ \mathbf{u}=\mathbf{g} & \text { on } \Gamma\end{cases}
$$

assorted with initial velocity conditions, where $\partial_{t}(\cdot)$ stands for partial differentiation with respect to time $t$ and $\llbracket q \rrbracket$ represents the jump across $\Sigma$ of the traces of a function $q \in H^{1}(\Omega \backslash \Sigma)$ on both sides of $\Sigma$. As usual $H_{0}^{1}(\Omega)$ is the subspace of $H^{1}(\Omega)$ of those functions that vanish on $\Gamma$ and $L_{0}^{2}(\Omega)$ is the subspace of $L^{2}(\Omega)$ consisting of functions $f$ such that $\int_{\Omega} f d x=0$.

In [13] we studied finite element approximations of (1) in the case where the inertia term $\rho \mathbf{u} \cdot \nabla \mathbf{u}$ is negligible as compared to the viscous term $\mu \Delta \mathbf{u}$. This corresponds to flow at a low Reynolds number, and in this case the Navier-Stokes system (1) reduces to a Stokes system, whose equivalent variational form can be stated as follows.

Denoting by $(\cdot, \cdot)$ the standard inner product of $L^{2}(\Omega)$ in scalar, vector or tensor version, and by $(\cdot, \cdot)_{D}$ the standard inner product of $L^{2}(D)$ for $D \subsetneq \Omega$, as long as $\mu$ is large enough so that inertia can be neglected and the flow is stationary, we may rewrite the underlying simplification of problem (1) in the following variational form of the Galerkin type:

$$
\begin{cases}\text { Find } \mathbf{u} \in\left[H^{1}(\Omega)\right]^{2} \text { and } p \in L_{0}^{2}(\Omega) & \text { such that } \\ \mu(\nabla \mathbf{u}, \nabla \mathbf{v})-(p, \operatorname{div} \mathbf{v})=(\mathbf{f}, \mathbf{v})+(\varphi, \mathbf{v} \cdot \boldsymbol{\nu})_{\Sigma} & \forall \mathbf{v} \in\left[H_{0}^{1}(\Omega)\right]^{2} \\ (\operatorname{div} \mathbf{u}, q)=0 & \forall q \in L_{0}^{2}(\Omega) \\ \mathbf{u}=\mathbf{g} & \text { on } \Gamma,\end{cases}
$$


where $\boldsymbol{\nu}$ is the unit normal vector on $\Sigma$ oriented from $\Omega_{1}$ towards $\Omega_{2}$, and the symbol . is used to represent the euclidean inner product of two vectors of $\mathbb{R}^{2}$.

Clearly enough, as a part of the solution of (2), the pressure $p$ is a priori discontinuous on $\Sigma$.

Observing that every $v \in H^{1}(\Omega)$ belongs to $L^{4}(\Omega)$ (cf. [10]), problem (2) can be easily modified into an equivalent variational form of (1), by adding the term $\rho(\mathbf{u} \cdot \nabla \mathbf{u}, \mathbf{v})$ to the right hand side of the first (momentum) equation.

\section{$3 \quad$ Finite element formulations}

\subsection{Preliminaries}

Let $\mathcal{P}$ be a quasi-uniform family of partitions (cf. [16]) $\mathcal{T}_{h}$ of $\Omega$ consisting of triangles with maximum edge length equal to $h$, and whose union is a polygonal approximation $\Omega_{h}$ of $\Omega$, in such a way that all of its vertices lie on $\Gamma$. We denote by $\Gamma_{h}$ the boundary of $\Omega_{h}$ which coincides with $\Gamma$ in case $\Omega$ is a polygon. The assumption that $\Omega$ be a convex domain in case it is not a polygon is further made in order to avoid some non essential complications related to external approximations. Of course this is an academic assumption, since our method works as well for non convex domains.

It is natural to approximate problem (2) using a pressure finite element space consisting of discontinuous functions on $\Sigma$. One could do this by matching the mesh in such a way that $\Sigma$ is approximated by the union of edges of a patch of elements in $\mathcal{T}_{h}$. However in doing so one would loose the possibility of working with structured or even uniform meshes, if $\Omega$ is rectangular for instance, since $\Sigma$ may be of very irregular shape. Therefore our numerical approach is based on a modification of the usual continuous piecewise linear finite element space to represent the pressure, incorporating discontinuities over $\Sigma$ (in fact, $\Sigma_{h}$ ) without requiring conforming meshes. The adopted space was originally proposed in [11] and thoroughly assessed in [14].

Before recalling the construction of such a space and introducing the finite element formulation, let us include necessary geometrical definitions and assumptions.

For numerical purposes $\Sigma$ will be approximated by a polygonal line $\Sigma_{h}$ constructed in connection with $\mathcal{T}_{h}$ in the following manner (see also Figure 1). Let $\mathcal{R}_{h}$ be the set of elements in $\mathcal{T}_{h}$ whose closures have a non empty intersection with $\Sigma$, and $\mathcal{S}_{h}$ be the subset of $\mathcal{R}_{h}$ such that $T \in \mathcal{S}_{h}$ means that there are two edges of $T$ cut by $\Sigma$ at least at one point different from their common vertex. Then $\Sigma_{h}$ is a polygonal approximation of $\Sigma$ formed by the chords joining two points of $\bar{T} \cap \Sigma$ on different edges of $T \in \mathcal{S}_{h}$ that are not vertices of $T$. We assume $\Sigma_{h}$ to have an orientation, and denote by $\boldsymbol{\nu}_{h}$ its oriented normal. In case $\Sigma_{h}$ divides $\Omega_{h}$ in two separate subdomains, these will be denoted as $\Omega_{h 1}$ and $\Omega_{h 2}$. In this case $\boldsymbol{\nu}_{h}$ is assumed to point from $\Omega_{h 1}$ towards $\Omega_{h 2}$.

With the above definitions of $\Sigma_{h}$, we assume that whenever $\Sigma$ is connected so is $\Sigma_{h}$. Actually this assumption will hold true provided some conditions on the mesh listed in [13] are satisfied. For instance the case where a vertex or an edge of the mesh is exactly contained in $\Sigma$ must be ruled out, by slightly perturbing the mesh (or $\Sigma$ ). We just recall here a basic condition concerning " $h$ being small enough".

Hypothesis H: The mesh size $h$ is small enough for the maximum absolute value of the curvature [15] of $\Sigma$ to be much smaller than $h^{-1}$. Moreover in case $\Omega$ is a polygon, $h$ is sufficiently small for the distance from $\Sigma$ measured along $\Gamma$ of anyone of its vertices to be greater than $h$.

Now if $T \in \mathcal{S}_{h}$, we subdivide the quadrilateral subset $K$ of $T$ lying on one side of $\Sigma_{h}$, into two triangles $T_{1}$ and $T_{2}$ by means of the diagonal of $K$ adjacent to its largest angle. We choose $T_{i}$ to be the triangle having $3-i$ vertices coinciding with vertices of $T, i=1,2$ (cf. Figure 2). 


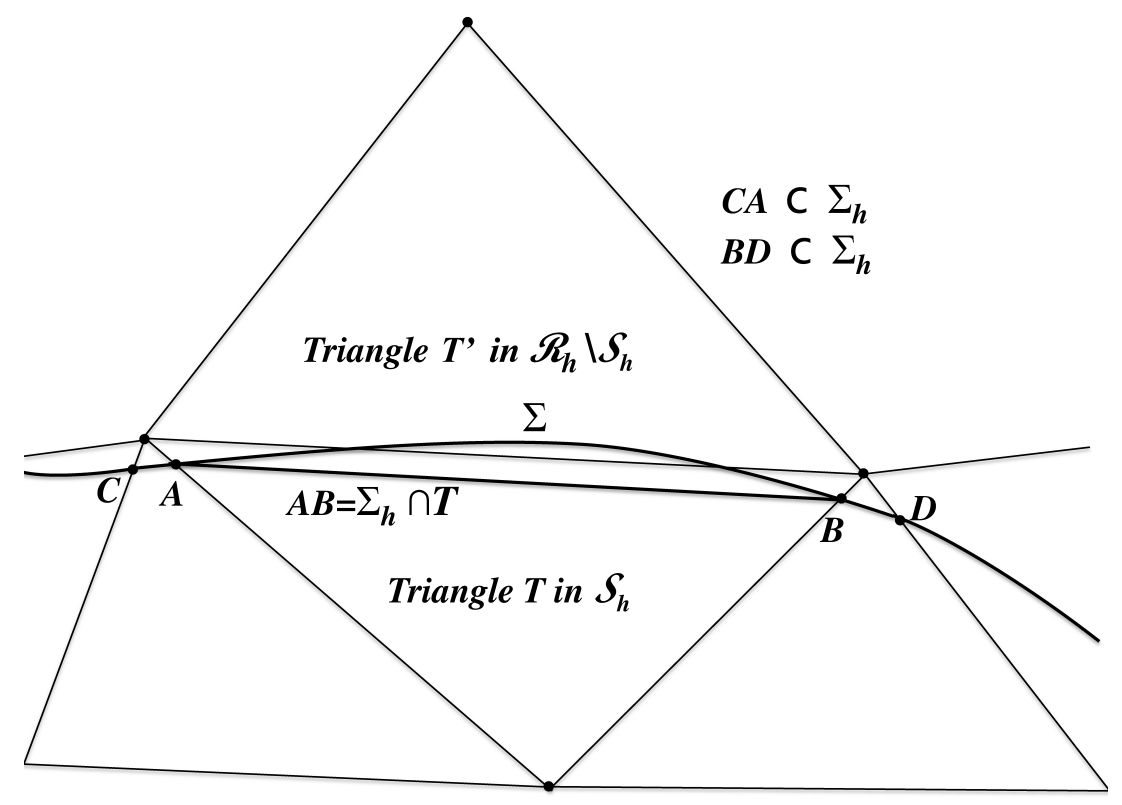

Figure 1. Local construction of $\Sigma_{h}$.

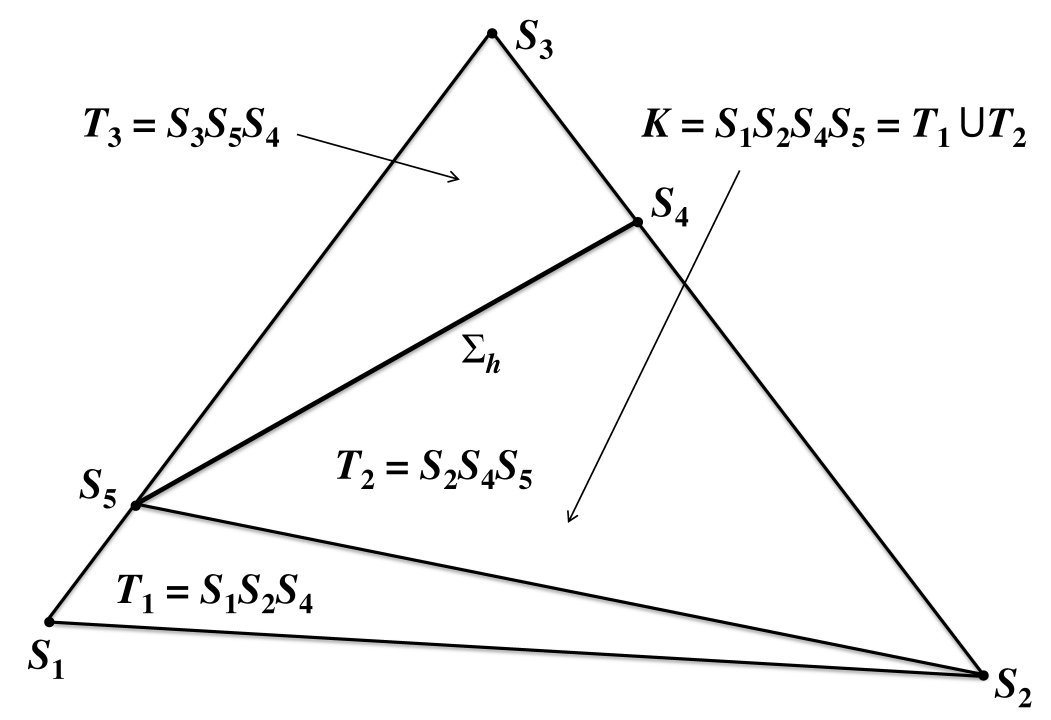

Triangle $T=S_{1} S_{2} S_{3}=K \cup T_{3}$

Figure 2. Sub-division of a triangle cut by $\Sigma_{h}$ into three triangles

The pressure space associated with $\mathcal{T}_{h}$ of discontinuous functions in the elements of $\mathcal{S}_{h}$, denoted here by $\tilde{Q}_{h}$, was introduced in [11]. For the sake of completeness we recall its definition as follows: Every function $q \in \tilde{Q}_{h}$ is necessarily continuous in $\Omega_{h} \backslash \Sigma_{h}$; in each triangle not belonging to $\mathcal{S}_{h}, q$ is either constant or 
linear. Referring to Figure 2 for a generic triangle $T \in \mathcal{S}_{h}, q_{\mid T_{3}}$ is constant, $q$ is linear in $T_{i}$ for $i=1,2$ and satisfies $q\left(S_{1}\right)=q\left(S_{5}\right)$ and $q\left(S_{2}\right)=q\left(S_{4}\right)$. An illustration of a set of basis functions for $\tilde{Q}_{h}$ in a triangle $T \in \mathcal{S}_{h}$ is supplied in Figure 3 .

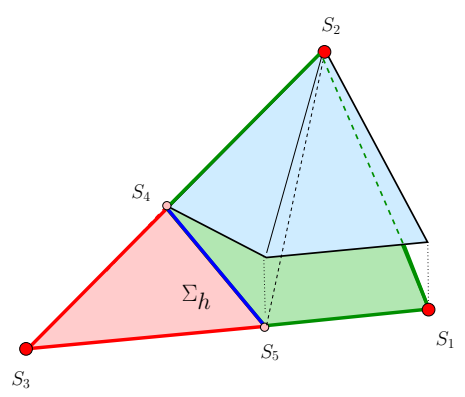

$q_{2}$

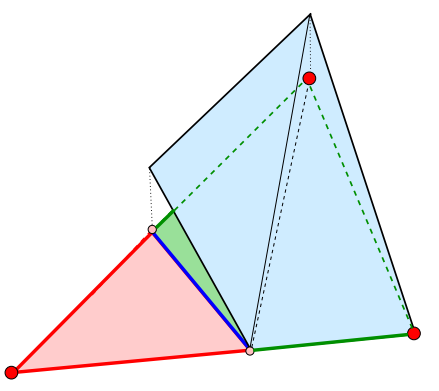

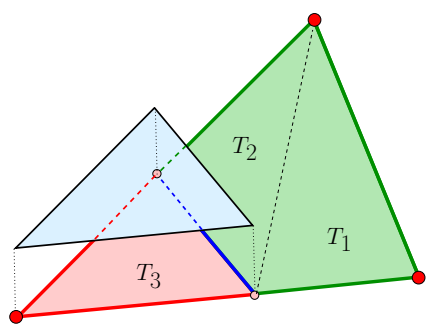

Figure 3. Set of basis functions $q_{i}, i=1,2,3$ for the space of discontinuous pressures $\tilde{Q}_{h}$ on a triangle $T \in \mathcal{S}_{h}$.

Henceforth the intersection of $\tilde{Q}_{h}$ with $L_{0}^{2}\left(\Omega_{h}\right)$ (cf. [19]) will be denoted by $Q_{h}$.

The velocity space $\mathbf{V}_{h}$ is the standard space of continuous fields whose restriction to each triangle in $\mathcal{T}_{h}$ is linear. We denote by $\mathbf{V}_{h 0}$ the subspace of $\mathbf{V}_{h}$ consisting of those fields that vanish identically on $\Gamma_{h}$.

\subsection{The proposed formulation for the Stokes case}

In the sequel $\mathbf{g}_{h}$ stands for the piecewise linear interpolate of $\mathbf{g}$ along $\Gamma_{h}$ at the vertices of $\mathcal{T}_{h}$ lying on $\Gamma$. Moreover we denote by $(\cdot, \cdot)_{m, D}$ the standard inner product of $H^{m}(D)$ and by $\|\cdot\|_{m, D}$ the associated norm. The subscript $m$ is frequently omitted when $m=0$.

Before recalling the solution method experimented in this work, we still have to define a piecewise gradient operator $\nabla_{\Sigma_{h}}$ of a function $q \in L^{2}\left(\Omega_{h}\right)$ such that $q \in H^{1}\left(\Omega_{h} \backslash \Sigma_{h}\right)$ as follows:

$$
\nabla_{\Sigma_{h}} q(\mathbf{x})=\left.\nabla q\right|_{\Omega_{h} \backslash \Sigma_{h}}(\mathbf{x}), \text { if } \mathbf{x} \in \Omega_{h} \backslash \Sigma_{h}
$$

Now given a strictly positive parameter $\delta$ and a non negative parameter $\varepsilon$, our finite-element approximation of (2) is as follows:

$$
\left\{\begin{array}{l}
\text { Find } \mathbf{u}_{h} \in \mathbf{V}_{h} \text { and } p_{h} \in Q_{h} \text { such that } \forall \mathbf{v} \in \mathbf{V}_{h 0} \text { and } \forall q \in Q_{h}, \\
\mu\left(\nabla \mathbf{u}_{h}, \nabla \mathbf{v}\right)_{\Omega_{h}}-\left(p_{h}, \operatorname{div} \mathbf{v}\right)_{\Omega_{h}}=\left(\mathbf{f}_{h}, \mathbf{v}\right)_{\Omega_{h}}+\left(\varphi_{h}, \mathbf{v} \cdot \boldsymbol{\nu}_{h}\right)_{\Sigma_{h}} \\
\varepsilon\left(\llbracket p_{h} \rrbracket_{h}, \llbracket q \rrbracket_{h}\right)_{\Sigma_{h}}+\delta\left(\nabla_{\Sigma_{h}} p_{h}, \nabla_{\Sigma_{h}} q\right)_{\Omega_{h}}+\left(\operatorname{div} \mathbf{u}_{h}, q\right)_{\Omega_{h}}=\varepsilon\left(\varphi, \llbracket q \varphi_{h}\right)_{\Sigma_{h}}+\delta\left(\mathbf{f}_{h}, \nabla_{\Sigma_{h}} q\right)_{\Omega_{h}} \\
\mathbf{u}_{h}=\mathbf{g}_{h} \text { on } \Gamma_{h} .
\end{array}\right.
$$

where $\llbracket \eta \rrbracket_{h}$ is the jump across $\Sigma_{h}$ of a function $\eta$ discontinuous on this manifold. In [13] the following well-posedness result was proven for problem (4):

Proposition 3.1 Under Hypothesis $\mathrm{H}$, if $\varepsilon>0$ problem (4) has a unique solution.

If $\varepsilon=0$, then (4) becomes (5), which also has a unique solution, as shown below. 
Proposition 3.2 Under Hypothesis H, given a strictly positive parameter $\delta$, the following problem is well-posed:

$$
\left\{\begin{array}{l}
\text { Find } \mathbf{u}_{h} \in \mathbf{V}_{h} \text { and } p_{h} \in Q_{h} \text { such that } \forall \mathbf{v} \in \mathbf{V}_{h 0} \text { and } \forall q \in Q_{h}, \\
\mu\left(\nabla \mathbf{u}_{h}, \nabla \mathbf{v}\right)_{\Omega_{h}}-\left(p_{h}, \operatorname{div} \mathbf{v}\right)_{\Omega_{h}}=\left(\mathbf{f}_{h}, \mathbf{v}\right)_{\Omega_{h}}+\left(\varphi_{h}, \mathbf{v} \cdot \boldsymbol{\nu}_{h}\right)_{\Sigma_{h}} \\
\delta\left(\nabla_{\Sigma_{h}} p_{h}, \nabla_{\Sigma_{h}} q\right)_{\Omega_{h}}+\left(\operatorname{div} \mathbf{u}_{h}, q\right)_{\Omega_{h}}=\delta\left(\mathbf{f}_{h}, \nabla_{\Sigma_{h}} q\right)_{\Omega_{h}} \\
\mathbf{u}_{h}=\mathbf{g}_{h} \text { on } \Gamma_{h} .
\end{array}\right.
$$

Proof. Assume that $\varphi, \mathbf{g}$ and $\mathbf{f}$ vanish everywhere in their respective definition domains. Since (5) is a linear problem we have to prove that corresponding $\mathbf{u}_{h}$ and $p_{h}$ vanish identically. Since $\mathbf{u}_{h} \in \mathbf{V}_{h 0}$ let us take in the first and second equations of (5) $\mathbf{v}=\mathbf{u}_{h}$ and $q=p_{h}$. Adding up the resulting relations we come up with,

$$
\mu\left\|\nabla \mathbf{u}_{h}\right\|_{\Omega_{h}}^{2}+\delta\left\|\nabla_{\Sigma_{h}} p_{h}\right\|_{\Omega_{h}}^{2}=0 .
$$

Since $\mathbf{u}_{h}=\mathbf{0}$ on $\Gamma$, we have $\mathbf{u}_{h} \equiv \mathbf{0}$. On the other hand (6) implies that $p_{h}$ is constant in each connected component of $\Omega_{h} \backslash \Sigma_{h}$. If $\Sigma_{h}$ does not separate $\Omega_{h}$ into two disjoint parts, $p_{h}$ is constant everywhere and thus $p_{h} \equiv 0$. If $\Sigma_{h}$ separates $\Omega_{h}$ into $\Omega_{h 1}$ and $\Omega_{h 2}$, then $p_{h}=c_{i}$ in $\Omega_{h i}$ for $i=1,2$, where $c_{1}$ and $c_{2}$ are constants. Since $p_{h} \in Q_{h}$ we have $c_{1}$ area $\left(\Omega_{h 1}\right)+c_{2}$ area $\left(\Omega_{h 2}\right)=0$. Moreover from the first equation of $(5)$ we have $\left(p_{h}, \operatorname{div} \mathbf{v}\right)_{\Omega_{h}}=0 \forall \mathbf{v} \in \mathbf{V}_{h 0}$. Applying the divergence theorem in both $\Omega_{h 1}$ and $\Omega_{h 2}$, taking into account that $\nabla p_{h} \equiv \mathbf{0}$ in both sub-domains, this trivially yields $\left|c_{1}-c_{2}\right|\left|\int_{\Sigma_{h}} \mathbf{v} \cdot \boldsymbol{\nu}_{h} d s\right|=0 \forall \mathbf{v} \in \mathbf{V}_{h 0}$. Clearly enough there exists at least one field $\mathbf{V}_{h 0}$ such that $\int_{\Sigma_{h}} \mathbf{v} \cdot \boldsymbol{\nu}_{h} d s \neq 0$. Therefore $c_{1}=c_{2}$ and thus $p_{h} \equiv 0$.

We have thus recalled and adapted the jump-stabilized formulation of [13] for the case under study. Under suitable assumptions, a priori error estimates applying to problem (4) were proved in [13] for $\delta \sim h^{2}$ and $\varepsilon \sim h$, as long as $\mathbf{f}, \mathbf{g}, \varphi$ and the exact solution of (2) are sufficiently smooth. More precisely, it holds that

$$
\left\|\nabla\left(\mathbf{u}-\mathbf{u}_{h}\right)\right\|_{\Omega_{h}}+\left\|p-p_{h}\right\|_{\Omega_{h}}+\left\|\llbracket \tilde{p}-p_{h} \rrbracket\right\|_{\Sigma_{h}} \leq C(\mathbf{u}, p, \mathbf{f}, \varphi) h,
$$

where $C(\mathbf{u}, p, \mathbf{f}, \mathbf{g}, \varphi)$ is a constant depending only on $\mathbf{u}, p, \mathbf{f}, \mathbf{g}$ and $\varphi$, and $\tilde{p}$ is a suitable projection of $p_{\mid \Sigma}$ onto $\Sigma_{h}$.

We now turn to the incorporation of inertial and transient effects by combining this formulation with the ASGS method for the Navier-Stokes equations.

\subsection{Combined Algebraic Subgrid Scale and Pressure Jump Stabilization}

In order to take into account the inertial term in the momentum equation with the method of the previous section it would be natural to adopt the following discrete formulation for $\delta>0$ and $\varepsilon \geq 0$ :

$$
\left\{\begin{array}{l}
\text { Find } \mathbf{u}_{h} \in \mathbf{V}_{h} \text { and } p_{h} \in Q_{h} \text { such that } \forall \mathbf{v} \in \mathbf{V}_{h 0} \text { and } \forall q \in Q_{h} \\
\mu\left(\nabla \mathbf{u}_{h}, \nabla \mathbf{v}\right)_{\Omega_{h}}+\rho\left(\mathbf{u}_{h} \cdot \nabla \mathbf{u}_{h}, \mathbf{v}\right)_{\Omega_{h}}-\left(p_{h}, \operatorname{div} \mathbf{v}\right)_{\Omega_{h}}=\left(\mathbf{f}_{h}, \mathbf{v}\right)_{\Omega_{h}}+\left(\varphi_{h}, \mathbf{v} \cdot \boldsymbol{\nu}_{h}\right)_{\Sigma_{h}} \\
\varepsilon\left(\llbracket p_{h} \rrbracket_{h}, \llbracket q \rrbracket_{h}\right)_{\Sigma_{h}}+\delta\left[\rho\left(\mathbf{u}_{h} \cdot \nabla \mathbf{u}_{h}, \nabla_{\Sigma_{h}} q\right)_{\Omega_{h}}+\left(\nabla_{\Sigma_{h}} p_{h}, \nabla_{\Sigma_{h}} q\right)_{\Omega_{h}}\right]+\left(\operatorname{div} \mathbf{u}_{h}, q\right)_{\Omega_{h}} \\
=\varepsilon\left(\varphi_{h}, \llbracket q \rrbracket_{h}\right)_{\Sigma_{h}}+\delta\left(\mathbf{f}_{h}, \nabla_{\Sigma_{h}} q\right)_{\Omega_{h}} \\
\mathbf{u}_{h}=\mathbf{g}_{h} \text { on } \Gamma_{h} .
\end{array}\right.
$$

The formulation above works well in general, however, it does not consider stabilization of the convective terms in the momentum equation. Moreover, it is also desirable to consider non-stationary situations that 
are relevant in several applications. To this end, we propose the following combination of pressure-jump stabilization with the ASGS formulation proposed by Codina et al 2001 [22]:

$$
\left\{\begin{array}{l}
\text { Find } \mathbf{u}_{h} \in \mathbf{V}_{h} \text { and } p_{h} \in Q_{h} \text { such that } \forall \mathbf{v} \in \mathbf{V}_{h 0} \text { and } \forall q \in Q_{h}, \\
\left(\mathcal{G}_{\mathbf{u}}, \mathbf{v}\right)_{\Omega_{h}}+\left(2 \mu \mathbf{D}\left(\mathbf{u}_{h}\right), \nabla \mathbf{v}\right)_{\Omega_{h}}-\left(p_{h}, \operatorname{div} \mathbf{v}\right)_{\Omega_{h}}+\sum_{T \in \mathcal{T}_{h}} \delta_{T}\left(\mathcal{G}_{\mathbf{u}}+\nabla_{\Sigma_{h}} p_{h}, \mathbf{u}_{h} \cdot \nabla \mathbf{v}\right)_{T}+ \\
+\sum_{T \in \mathcal{T}_{h}} \alpha_{T}\left(\operatorname{div} \mathbf{u}_{h}, \operatorname{div} \mathbf{v}\right)_{T}-\left(\varphi_{h}, \mathbf{v} \cdot \boldsymbol{\nu}_{h}\right)_{\Sigma_{h}}=0 \\
\left(q, \operatorname{div} \mathbf{u}_{h}\right)_{\Omega_{h}}+\sum_{T \in \mathcal{T}_{h}} \frac{\delta_{T}}{\rho}\left(\mathcal{G}_{\mathbf{u}}+\nabla_{\Sigma_{h}} p_{h}, \nabla_{\Sigma_{h}} q\right)_{T}+\sum_{T \in \mathcal{T}_{h}} \varepsilon_{T}\left(\llbracket p_{h} \rrbracket-\varphi_{h}, \llbracket q_{h} \rrbracket\right)_{\Sigma_{h} \cap T}=0
\end{array}\right.
$$

where we have defined $\mathcal{G}_{\mathbf{u}}=\rho\left(\partial_{t} \mathbf{u}_{h}+\mathbf{u}_{h} \cdot \nabla \mathbf{u}_{h}\right)-\mathbf{f}_{h}$ and

$$
\begin{aligned}
\delta_{T} & =\left[4 \frac{\mu}{\rho h_{T}^{2}}+2 \frac{\left\|\mathbf{u}_{h}\right\|}{h_{T}}\right]^{-1}, \\
\alpha_{T} & =2 \mu+\rho\left\|\mathbf{u}_{h}\right\| h_{T}, \\
\varepsilon_{T} & =C_{\varepsilon} \frac{\delta_{T}}{\rho h_{T}}
\end{aligned}
$$

in which $h_{T}$ is the diameter of element $T$. Regarding this formulation the following observations are in order:

- Inertial and transient effects are now considered. The formulation is a natural extension of the singlephase ASGS formulation, which contains penalty terms for the momentum equation and for the continuity equation, respectively multiplied by $\delta_{T}$ and $\alpha_{T}$. The proposed modification is a third penalty term, this time for the interface equilibrium equation $\llbracket p \rrbracket=\varphi$.

- Notice that some simplifications have been made in the discrete formulation by particularizing it to (a) piecewise linear $\mathbf{u}_{h}$, and (b) constant viscosity. If the viscosity exhibits a jump through $\Sigma_{h}$, then the penalty term would also contain the jump of normal viscous stress across $\Sigma_{h}$.

- Elementwise penalty parameters $\delta_{T}$ and $\alpha_{T}$ are adopted, with the expressions suggested by Codina et al[22]. Similarly, an elementwise $\varepsilon_{T}$ is implemented and given an expression with consistent units, with a dimensionless constant $C_{\varepsilon}$ whose ideal value found by means of our numerical experimentation is 2 .

- Instead of using $\nabla \mathbf{u}_{h}$, the symmetric gradient $\mathbf{D}\left(\mathbf{u}_{h}\right)=\frac{1}{2}\left(\nabla \mathbf{u}_{h}+\nabla^{T} \mathbf{u}_{h}\right)$ appears in the momentum residual equation. This is needed for problems with Neumann boundary conditions and/or variable viscosity.

- In problem (9) the time step index has been dropped in all unknown fields to simplify the notation. As for time discretization, this problem is solved at each step for the unknowns $\mathbf{u}_{h}$ and $p_{h}$ in a coupled manner, using a Newton-Raphson iterative procedure together with the approximation

$$
\partial_{t} \mathbf{u}_{h} \approx \frac{\mathbf{u}_{h}-\mathbf{u}_{h}^{n}}{\Delta t}
$$

where $\Delta t$ is the time step and $\mathbf{u}_{h}^{n}$ stands for the velocity field at the last computed step.

Clearly, all formulations discussed in the previous section are particular cases of (9). For instance, in a stationary case, neglecting inertia and taking $\alpha_{T}=0$, we recover formulation (4). 


\section{Numerical experiments}

In this section we numerically assess the proposed discrete formulation with a few examples. We first consider the laminar flow in a horizontal channel in the presence of an actuator disk with several configurations and assess the convergence properties of the method. More complex configurations, considering inertial and transient effects, are also addressed. To show the versatility of the method, we finally solve a problem of interest in microfluidics, namely the capillary migration of a circular droplet with variable surface tension coefficient.

\subsection{Actuator disks - Stokes regime}

Let us consider the computational domain $[0, L] \times[0, H]$. A horizontal singular force $\varphi$ is applied along a straight vertical line defined by

$$
\Sigma_{h}=\left\{\left(x_{1}, x_{2}\right), x_{1}=a, x_{2} \in[b, H]\right\}
$$

This is the simplest actuator disk model that can be considered. Periodic boundary conditions are assumed in the $x_{1}$-direction and no-slip boundary conditions are taken on the bottom and top walls of the channel, i.e.,

$$
\mathbf{u}\left(x_{1}, x_{2}=0\right)=\mathbf{u}\left(x_{1}, x_{2}=H\right)=0
$$

The indeterminacy of the pressure is removed by imposing $p(0,0)=0$. An illustration of the computational domain is shown in Figure 4. Two cases are considered depending on the value adopted for $b$ in (14), namely:

- Case I - $b=0$ : the interface $\Sigma$ occupies the entire channel section. The normal force $\varphi$ is taken equal to 1 along $\Sigma$. The exact solution is known in this academic case and corresponds to a Poiseuille flow with quadratic velocity profile. The pressure field is piecewise linear exhibiting a jump equal to the value of $\varphi$ at the location of the interface $x_{1}=a$. This problem has been solved several times before (see e.g. [11,24]) and its solution reads

$$
\begin{aligned}
u_{1}\left(x_{1}, x_{2}\right) & =\frac{1}{2 \mu L}\left(H-x_{2}\right) x_{2}, \\
u_{2}\left(x_{1}, x_{2}\right) & =0 \\
p\left(x_{1}, x_{2}\right) & =-\frac{x_{1}}{L}+\mathcal{H}\left(x_{1}-a\right)
\end{aligned}
$$

where $\mathcal{H}(\cdot)$ is the Heaviside function (i.e., $\mathcal{H}(s)=1$ if $s>0$ and zero otherwise). A sequence of computational meshes is built of which the first one is shown in Figure 4. A characteristic mesh size $h=0.05$ is assigned to this mesh. The other meshes in the sequence are obtained by subdivision of the triangles into four identical triangles. As one can observe, the interface is neither conforming nor parallel to the edges of the finite element partition. The problem is solved taking $L=3, H=1, \mu=1$ and $a=2$. To illustrate the effect of the interface stabilization and the value adopted for the stabilization parameter $\left(C_{\varepsilon}=2\right)$, we compare results to a case with $C_{\varepsilon} \gg 1$ and a case with $C_{\varepsilon} \ll 1$. Figure 5 shows a section of the pressure field $p_{h}$ along the horizontal line at $x_{2}=H / 2$ corresponding to the first mesh in the sequence in the three cases. By watching the insert, we clearly observe the formulation performs best for the proposed choice of stabilization parameter, whereas choosing the stabilization parameter being very large or very small has a deleterious effect in the pressure field in the proximity of the interface. Notice that this occurs not only in the sense being observed, i.e. the mean-square $\left(L^{2}\right)$ sense, but also to a lesser extent in the pointwise $\left(L^{\infty}\right)$ sense. 
We report in Table 1 the $L^{2}\left(\Omega_{h}\right)$ and $H^{1}\left(\Omega_{h}\right)$ error norms of pressure and velocity, respectively, and an estimation of the convergence rates. The pressure error is close to an $O\left(h^{1.5}\right)$, which can be viewed as a super-convergence phenomenon, for both the unstabilized and the interface stabilized methods. However, the error is significantly smaller for the latter.

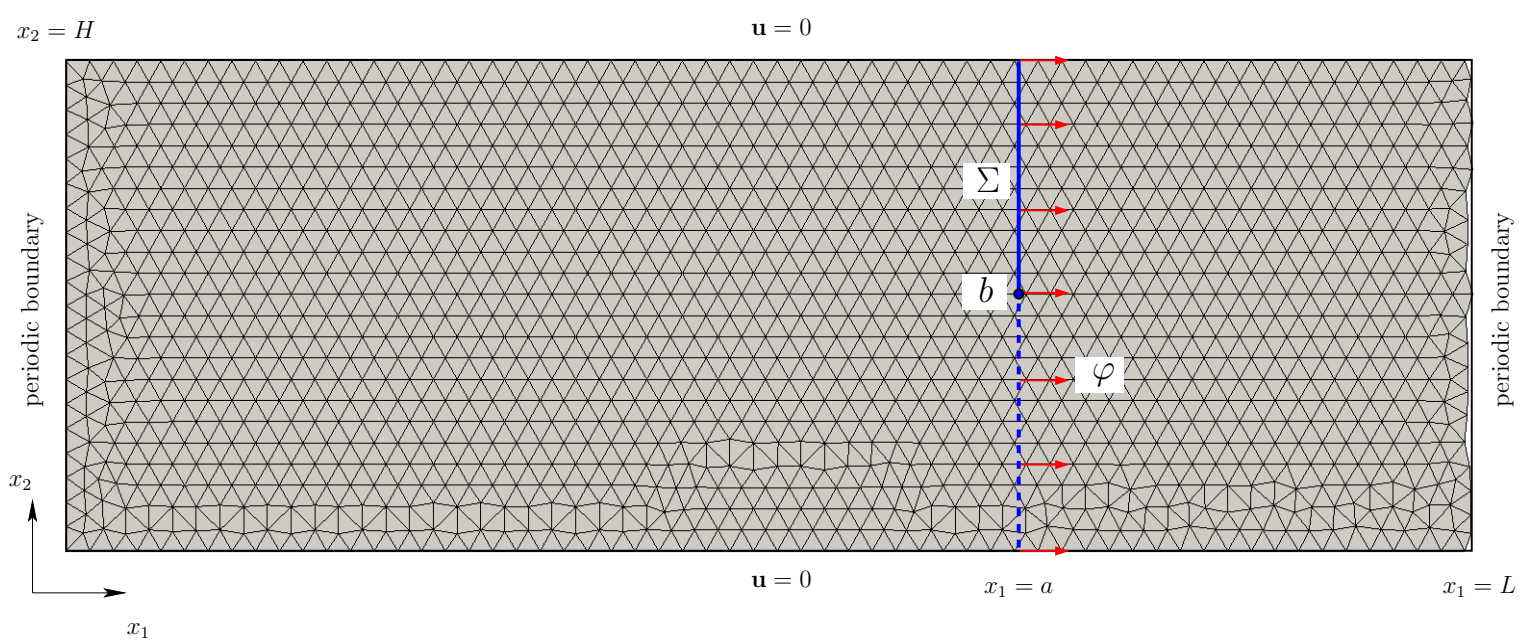

Figure 4. Setting for the actuator disk problem and the coarsest mesh used for discretization.

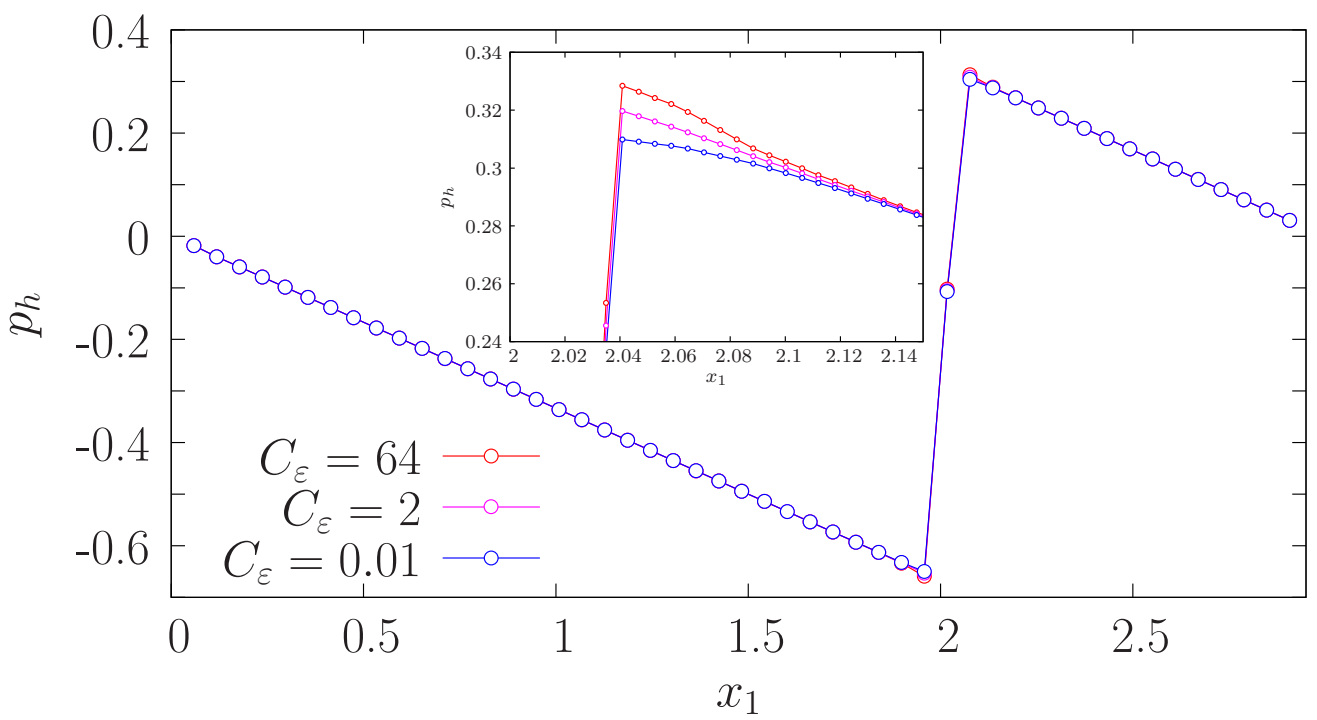

Figure 5. Section of pressure field along the line $x_{2}=H / 2=0.5$ in Case $\mathbf{I}$ of the actuator disk problem at the coarsest mesh level considering the overstabilized case $\left(C_{\varepsilon} \gg 1\right)$, the understabilized case $\left(C_{\varepsilon} \ll 1\right)$ and the proposed value $C_{\varepsilon}=2$.

- Case II - $b=0.5$ : this corresponds to an interface $\Sigma_{h}$ that occupies only half of the channel section. The same physical parameters and computational meshes of the previous case are used. The solution is not explicitly known in this case. The error is now measured by comparison of the solution for a given mesh to the solution obtained at the subsequent level of mesh refinement. 
Table 1

Error norms for pressure and velocity in Case I (interface $\Sigma$ occupying the entire channel section) of the actuator disk problem.

\begin{tabular}{r|cc|cc} 
& \multicolumn{2}{c}{ Unstabilized } & \multicolumn{2}{c}{ Stabilized } \\
& $\left\|p-p_{h}\right\|_{0, \Omega_{h}}$ & $\left\|\mathbf{u}-\mathbf{u}_{h}\right\|_{1, \Omega_{h}}$ & $\left\|p-p_{h}\right\|_{0, \Omega_{h}}$ & $\left\|\mathbf{u}-\mathbf{u}_{h}\right\|_{1, \Omega_{h}}$ \\
\hline$h / 4$ & 0.00043 & 0.00181 & 0.000134 & 0.00181 \\
$h / 2$ & 0.00123 & 0.00383 & 0.000357 & 0.00362 \\
$h$ & 0.00353 & 0.00727 & 0.00096 & 0.00724 \\
Order & 1.515 & 1.002 & 1.424 & 1.000
\end{tabular}

As for the force to be applied over $\Sigma_{h}$, in the actuator disk model, the ideia is to replace the effect of a real propeller with a permeable disk over which the forces acting on the fluid are distributed. In order to qualitatively mimic this force the following variation is chosen for $\varphi$ (see e.g. [1,25])

$$
\varphi=c\left(1-x_{2}\right) \tanh \left[10\left(x_{2}-0.5\right)\right]
$$

where the factor $c$ is set equal to 3.1344 such that the maximum value of $\varphi$ approximately equals 1 . Pressure and velocity fields are illustrated in Figure 6. In Figure 7 the pressure jump and the applied force are plotted for the coarsest mesh level, comparing the stabilized and unstabilized cases. As one can infer from this figure, the interface stabilized formulation allows for a much better representation of the discontinuity in the pressure field on $\Sigma_{h}$. Table 2 reports the $L^{2}\left(\Omega_{h}\right)$ and $H^{1}\left(\Omega_{h}\right)$ error norms for pressure and velocity respectively and the convergence rate in both cases. It should be noticed that the estimated rate of convergence is ca. 1.3, which is closer to the theoretical prediction of $O(h)$, than the super-convergence rate of ca. 1.5 observed in the previous case. However, both are quite similar, which means that the use of the interface stabilization does not have any deleterious effect on the results considering these global norms. On the other hand, through the error measure of the jump, the advantage of the new formulation clearly appears as in the previous case.

Table 2

Error norms for pressure and velocity in Case II (interface $\Sigma$ occupying half of the channel section) of the actuator disk problem.

\begin{tabular}{r|cc|cc} 
& \multicolumn{2}{c}{ Unstabilized } & \multicolumn{2}{c}{ Stabilized } \\
& $\left\|p-p_{h}\right\|_{0, \Omega_{h}}$ & $\left\|\mathbf{u}-\mathbf{u}_{h}\right\|_{1, \Omega_{h}}$ & $\left\|p-p_{h}\right\|_{0, \Omega_{h}}$ & $\left\|\mathbf{u}-\mathbf{u}_{h}\right\|_{1, \Omega_{h}}$ \\
\hline$h / 8$ & - & - & - & - \\
$h / 4$ & 0.00223 & 0.00216 & 0.00219 & 0.00214 \\
$h / 2$ & 0.00521 & 0.00424 & 0.00546 & 0.00417 \\
$h$ & 0.01341 & 0.00829 & 0.02131 & 0.00814 \\
Order & 1.223 & 0.980 & 1.318 & 0.962
\end{tabular}

\subsection{Actuator disks - Inertial and transient effects}

\section{Stationary case}

The problem setting considered is shown in Figure 8 with two inclined actuator disks positioned under a square obstacle. In this case we opt to provide all quantities and results in dimensional form. The computational domain has width $L=10 \mathrm{~cm}$ and height $H=20 \mathrm{~cm}$ and the obstacle is a square of size 2 $\mathrm{cm}$ (see Figure 8). Fluid properties correspond to that of air, with density $\rho=1.2 \mathrm{Kg} \cdot \mathrm{m}^{-3}$ and viscosity 

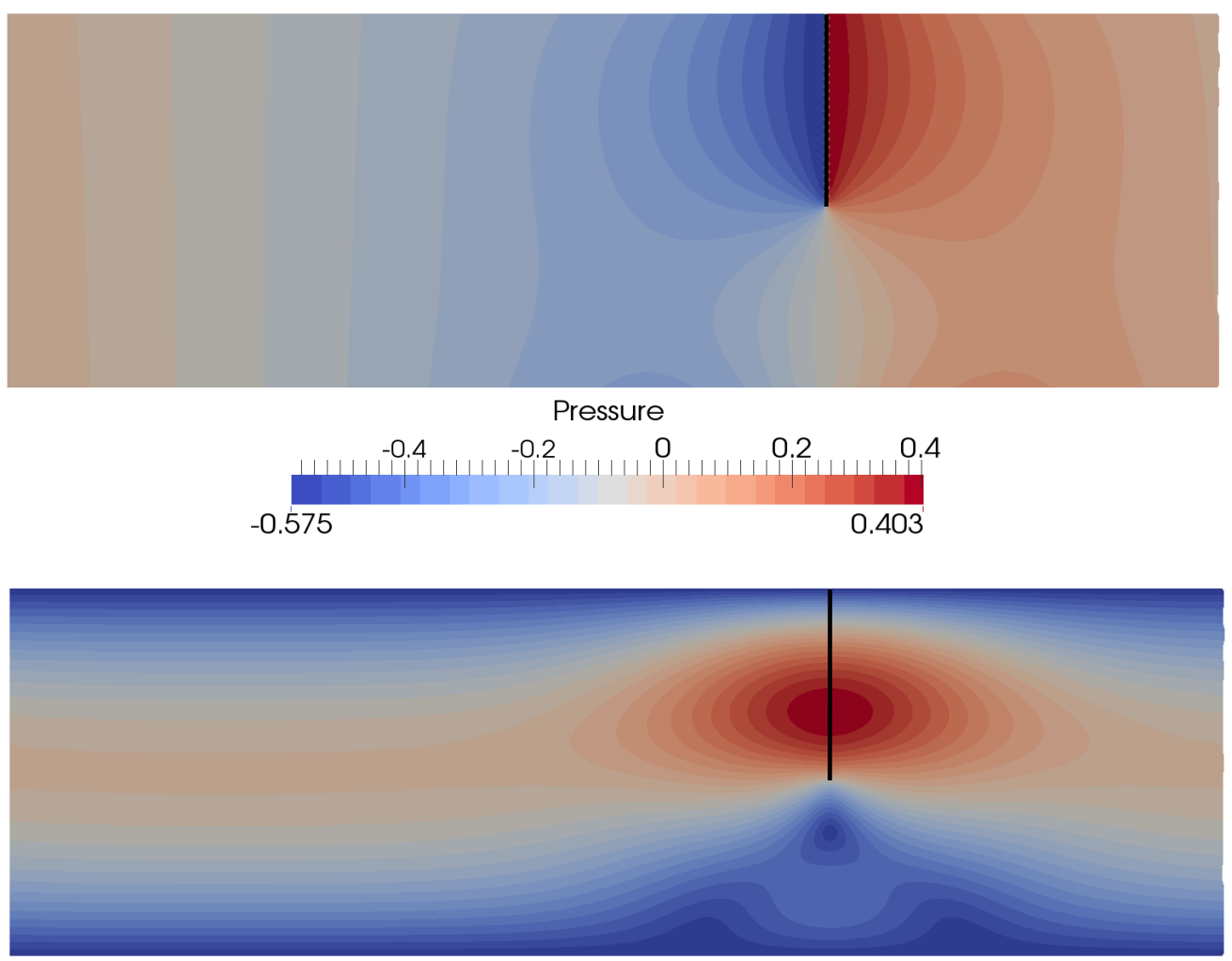

Velocity Magnitude

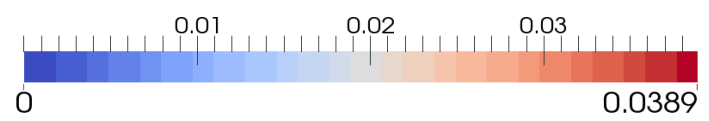

Figure 6. Pressure and velocity field in Case II of the actuator disk problem for the second mesh level.

$\mu=2 \times 10^{-5} \mathrm{~Pa} \cdot \mathrm{s}$. On the lateral sides of the domain no-slip boundary conditions are imposed. The bottom and top walls are respectively inlet and outlet boundaries, so the fluid is free to enter or leave the domain, however, at the outlet the horizontal component of the velocity field is set to zero. The domain is discretized with a mesh also shown in Figure 8 having 18,042 triangles and a characteristic mesh size $h=0.065$ near the actuator disks. Over both disks a normal force given by

$$
\varphi(s, t)= \begin{cases}C(t) s \tanh [4(0.5-s)] & \text { if } s<0.5 \\ C(t)(1-s) \tanh [4(s-0.5)] & \text { if } s \geq 0.5\end{cases}
$$

is applied, where for each disk $s \in[0,1]$ is a line parameter along the horizontal direction as indicated in figure 8 and $C$ is used to control the magnitude of this force as a function of time. This way, one is able to produce some complex flow patterns around the obstacle and test the proposed method in a more challenging situation in the presence of both, inertial and transient effects. Notice that testing other geometrical configurations (disk's size, position and inclination) would very simple using this unique background mesh. We look for a steady state solution by taking a very large time step. Figure 9 shows pressure and velocity for the simulated flow field. These results correspond to a constant value of $C$ in 


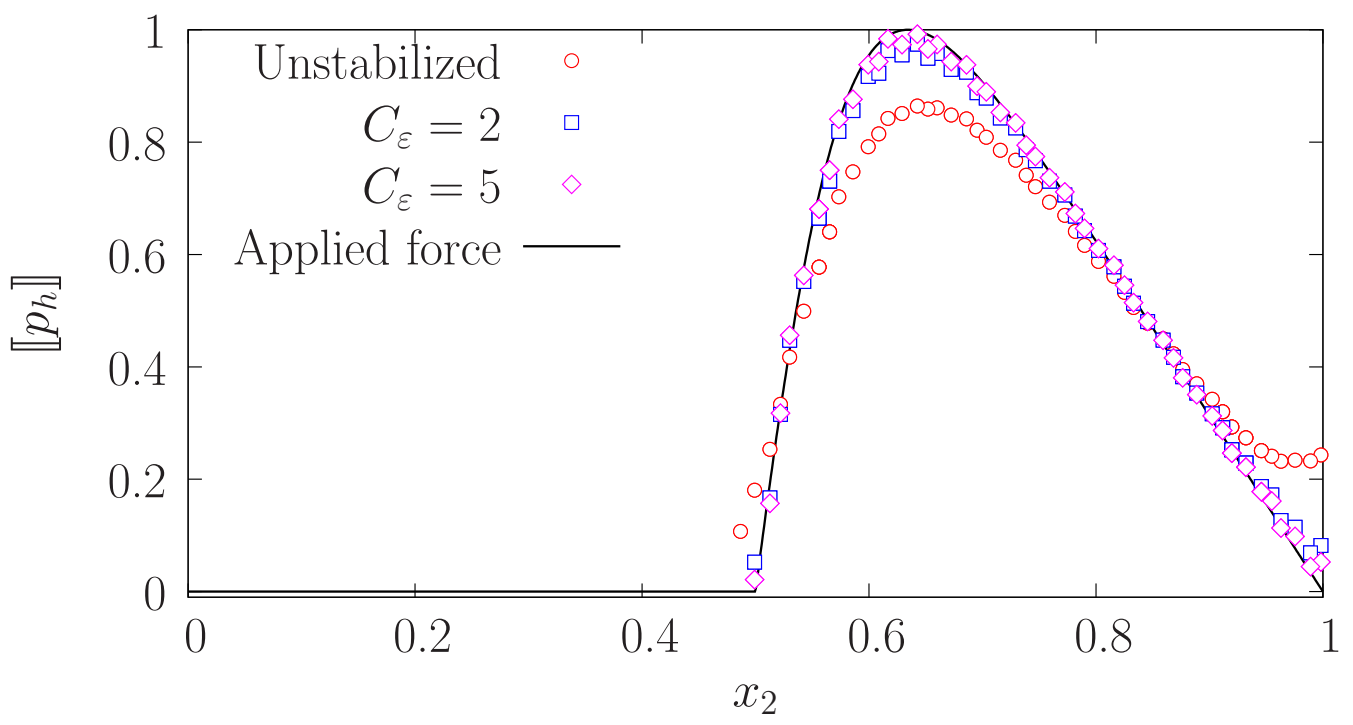

Figure 7. Singular force and pressure jump along $\Sigma$ in Case II of the actuator disk problem for the interface stabilized and unstabilized case at the second refinement level.

(20) equal to $3.85 \times 10^{-4} \mathrm{~Pa}$ and the Reynolds number in this case is ca. 22.5 based on the obstacle length scale, that is $2 \mathrm{~cm}$. The complex flow pattern in the present case is noteworthy, as compared to the previous simulations. Figure 10 shows the applied force and compares it to the numerical pressure jump along the interface for the stabilized and unstabilized formulations. These results certify again, the superior behavior of the former. It is important to note that the velocity field does not exhibit any significant difference with respect to the unstabilized formulation as indicated in Figure 11 where the magnitude of velocity $\left\|\mathbf{u}_{h}\right\|_{2}$ is plotted along the left interface and compared to the solution obtained with a fine grid having characteristic mesh size $h=0.02$ near $\Sigma_{h}$, that is, 3.25 times a smaller mesh size than the one of the orginal mesh showed in Figure 8. We have observed comparable results for other geometrical configurations of the actuator disks.

\section{Transient case - Fixed disks}

In the transient case the behavior is analogous. For illustration we progressively increased the amplitude $C$ of the force (20) from the value $3.13 \times 10^{-5}$ to $5.95 \times 10^{-3}$ Pa for $0 \leq t \leq 1 \mathrm{~s}$. From $t=1 \mathrm{~s}$, the amplitude of $\varphi$ remains constant at the value $C(t=1)$. Results are presented in Figure 12 where pressure and velocity contours are displayed at three different times, namely, $t=1,3$ and $5 \mathrm{~s}$. The top part of Figure 13 shows the applied force and pressure jump for the stabilized and unstabilized formulations along the left actuator disk at two different times, namely, $t=0.5$ and $t=4.5$. Similar results are observed for the right disk and also at other times along the simulation. The bottom part of this figure shows the magnitude of the velocity field $\left\|\mathbf{u}_{h}\right\|_{2}$ along the interface for the values of $t$. Here again we observe the velocity along $\Sigma_{h}$ produced by the stabilized and unstabilized methods are very similar.

\section{Transient case - Moving disk}

We finally consider the geometrical setting shown in Figure 14. The computational domain is the square region $[0,10] \times[0,10] \mathrm{cm}$. A rotating disk of length $4 \mathrm{~cm}$ is placed at the center. Figure 14 shows the disk at several inclination angles, namely, $\pi / 16$ (maximum inclination), 0 and $-\pi / 16$ (minimum inclination). The angular velocity $\omega$ of the disk is prescribed accordingly by 


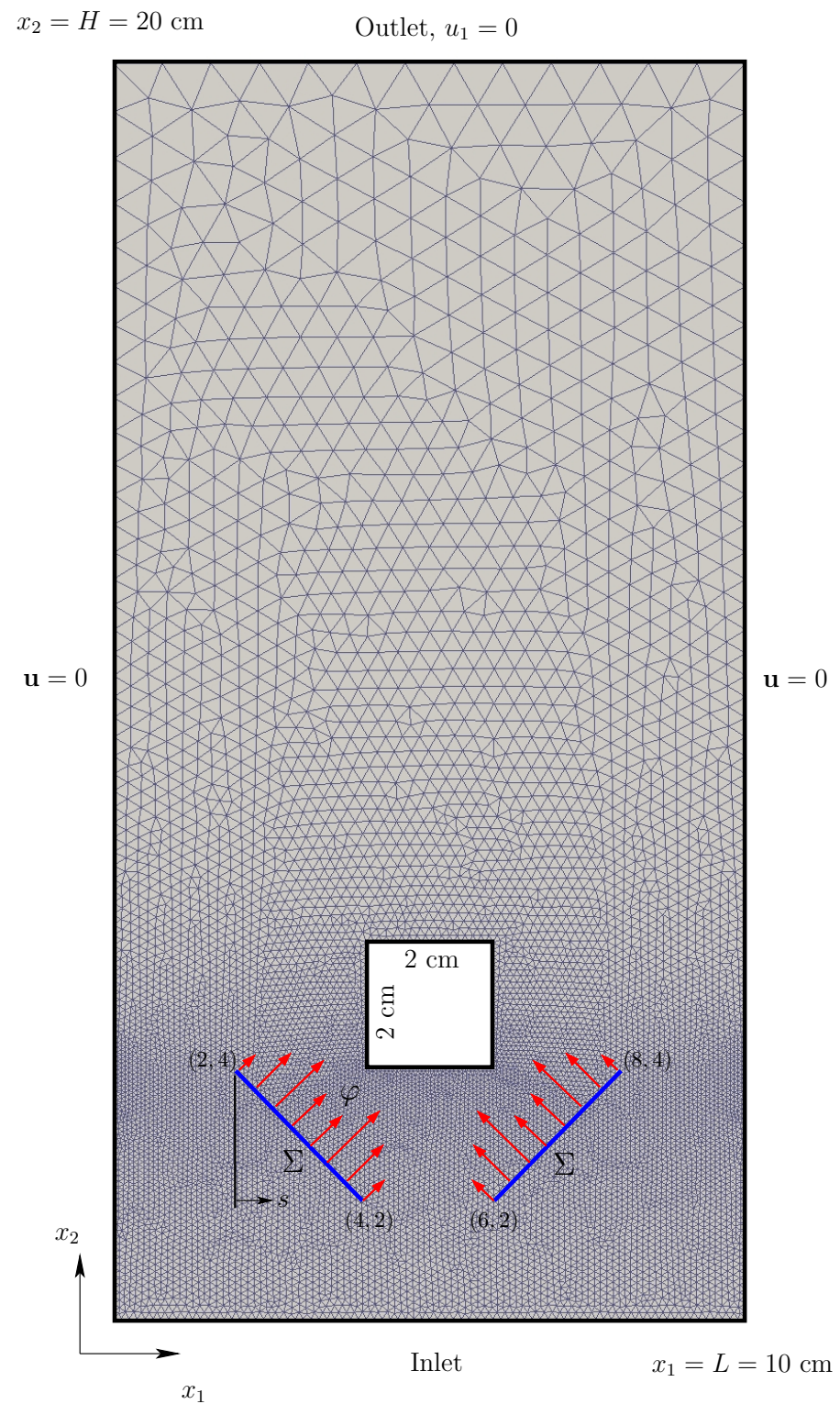

Figure 8. Problem setting to study the influence of two inclined actuator disks on the flow around a square obstacle taking into account inertial and transient effects.

$$
\omega(t)=\frac{5 \pi^{2}}{8} \cos (10 \pi t)
$$

The prescribed force is similar to previous cases

$$
\varphi(s, t)= \begin{cases}-C(t)(s+0.5) \tanh (4 s) & \text { if } s<0.5 \\ C(t)(s-0.5) \tanh (4 s) & \text { if } s \geq 0.5\end{cases}
$$



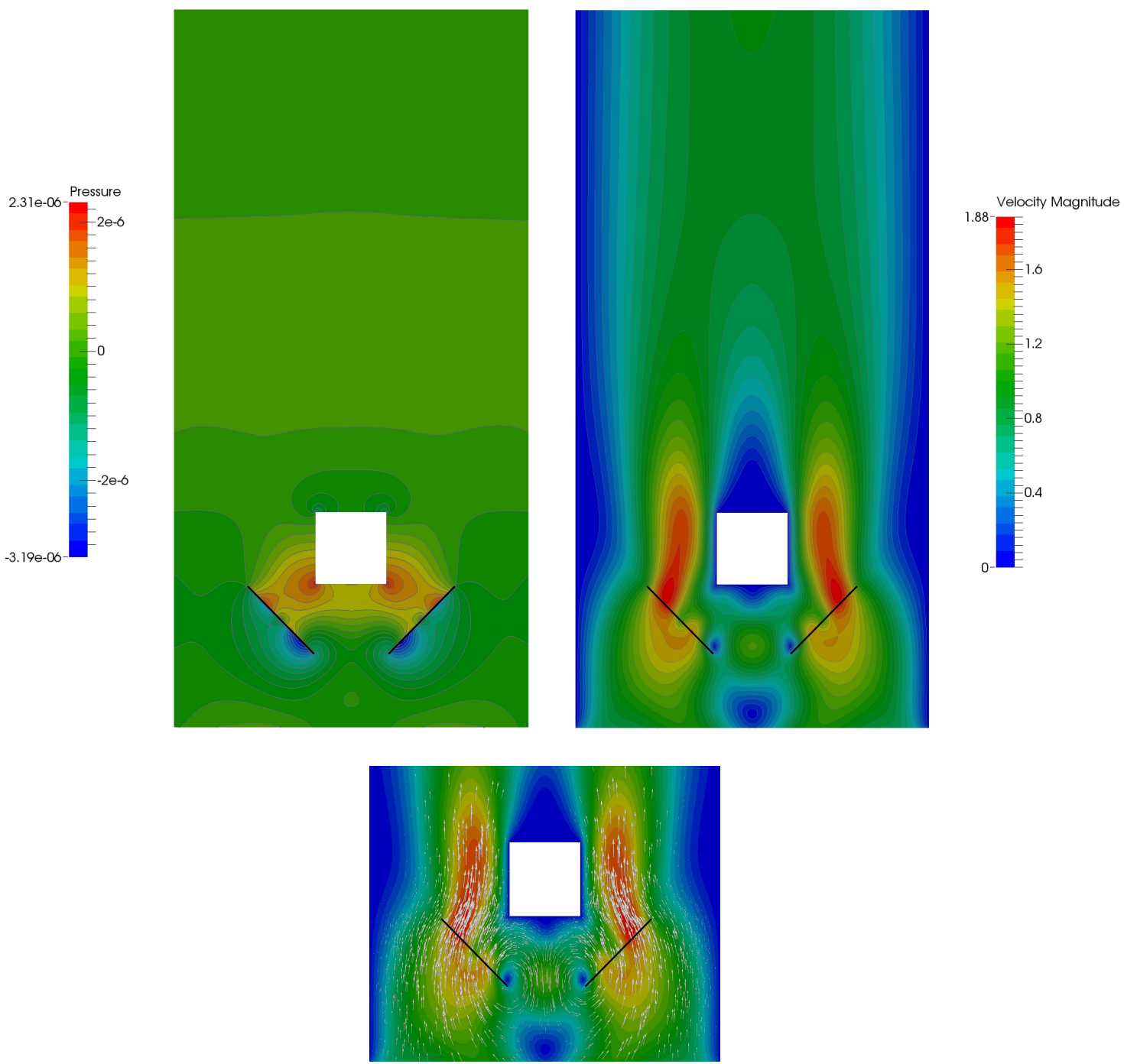

Figure 9. Pressure field (in $\left.\mathrm{Kg} /\left(\mathrm{cm} \cdot \mathrm{s}^{2}\right)\right)$ and velocity field (in $\mathrm{cm} / \mathrm{s}$ ) illustrating the influence of two inclined actuator disks inducing stationary flow around a square obstacle at a Reynolds number $R e \approx 22.5$.

except that now $s \in[-0.5,0.5]$ is a line parameter along the interface being equal to 0 at the disk's center. The amplitude $C(t)$ is taken as

$$
C(t)=a \operatorname{sign}[\omega(t)]
$$

where $a$ is a parameter to be chosen. Notice that with this choice for $C(t)$ the force produced by the disk instantaneously changes sign in the same way $\omega(t)$ does. Pressure and velocity fields at different times are shown in figure 15 corresponding to the stabilized formulation and the value $a=2 \times 10^{-3} \mathrm{~Pa}$ in (23). The same physical parameters of the previous numerical experiment are considered. The time step $\Delta t$ is set to $0.33 \times 10^{-3} \mathrm{~s}$. Based on the disk's length, the Reynolds number for this problem ranges between 700 and 2000. From figure 15 we indeed observe more complex flow patterns as compared to previous numerical simulations. It is interesting to plot in this problem the pressure jump error as a function of time for the stabilized and unstabilized formulations. The top part of figure 16 shows the error for $a=2 \times 10^{-4}$ and 


\section{Unstabilized ○ \\ Stabilized $-C_{\varepsilon}=5 \quad \circ$ \\ Applied force}

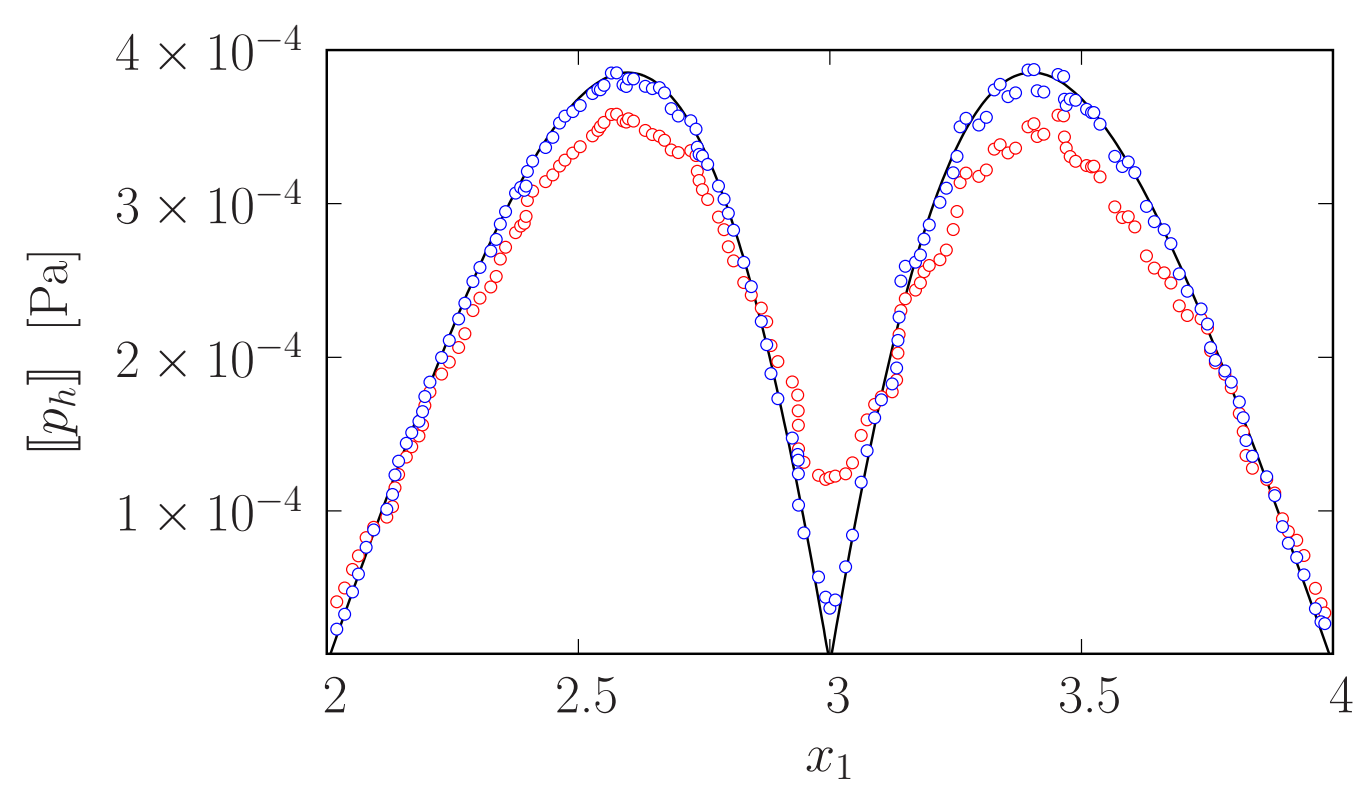

Figure 10. Pressure jump and applied force along the left interface for the actuator disks inducing stationary flow around a square obstacle by considering inertia.

the bottom part shows results for $a=2 \times 10^{-3} \mathrm{~Pa}$ (the same value that corresponds to the contours showed in figure 15). For each plot, the mean value, standard deviation (std) and maximum values over time of these errors are indicated. A superior behavior of the stabilized formulation at almost all times is observed, except for a very few times when $a=2 \times 10^{-3} \mathrm{~Pa}$.

From this numerical evidence we conclude that the proposed stabilization with the ASGS formulation allows to better capture pressure discontinuities on immersed interfaces in much more general situations than those originally considered in [12] and [13].

\subsection{Thermocapillary Marangoni migration of a droplet}

To illustrate the versatility of the method we finally move to an application of interest in micro-fluidics which is the Marangoni migration of a spherical immiscible droplet induced by a varying surface tension coefficient. In practice, such a variation may be the result of heterogeneities in the temperature field or the concentration of surfactant agents. Inertial effects are neglected, which is a reasonable assumption due to the typically small size of the system. The problem to be solved reads 


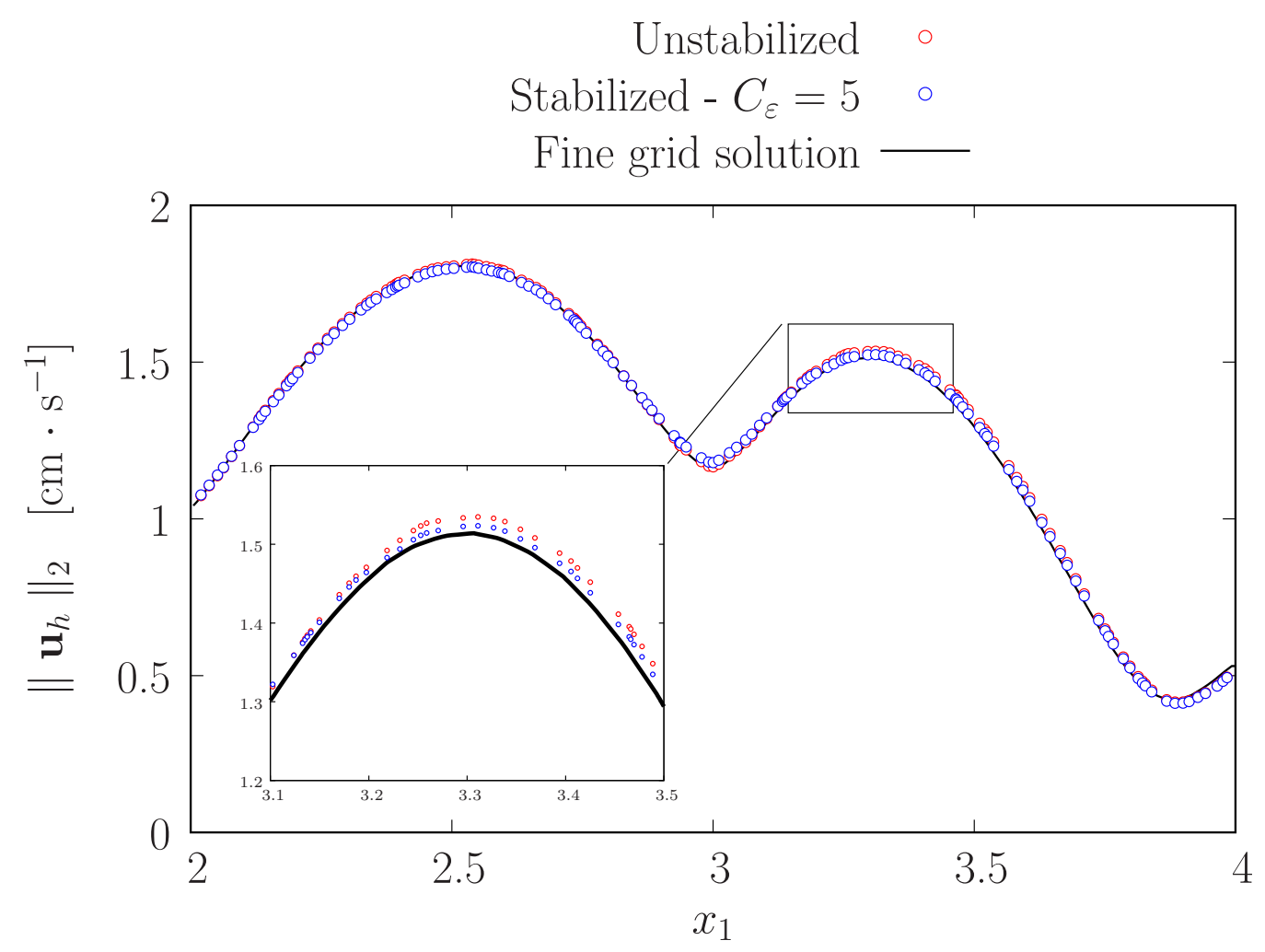

Figure 11. Magnitude of velocity field $\left\|\mathbf{u}_{h}\right\|_{2}$ on the left interface for the actuator disks inducing stationary flow around a square obstacle by considering inertia.

$$
\begin{cases}-\mu \Delta \mathbf{u}+\nabla p=0 & \text { in } \Omega \backslash \Sigma \\ \operatorname{div} \mathbf{u}=0 & \text { in } \Omega \\ \llbracket \boldsymbol{\sigma} \rrbracket \cdot \boldsymbol{\nu}=\mathbf{f}_{\Sigma} & \text { on } \Sigma \\ \mathbf{u}(\mathbf{x} \rightarrow \infty)=0, \quad p(\mathbf{x} \rightarrow \infty)=p_{\infty}, & \end{cases}
$$

When the surface tension coefficient $\gamma$ depends on $\mathbf{x}$, the singular force is given by

$$
\mathbf{f}_{\Sigma}=-\gamma \kappa \boldsymbol{\nu}+\nabla_{S} \gamma
$$

where $\kappa$ is the mean curvature and $\nabla_{S} \gamma=\mathbf{P} \cdot \nabla \gamma$ stands for the surface gradient of $\gamma, \mathbf{P}(:=\mathbf{I}-\boldsymbol{\nu} \otimes \boldsymbol{\nu})$ being the tangent projector onto $\Sigma$. The normal component of the singular force is thus $\varphi=-\gamma \kappa$. The force in (25) has also a non-zero tangential component leading to a jump of the velocity gradient on $\Sigma$. A linear variation of the surface tension coefficient is assumed, namely,

$$
\gamma=\gamma_{0}-\gamma_{1} x_{1}
$$

where $\gamma_{0}$ and $\gamma_{1}$ are constants. Therefore, the tangential component of the singular force equals $\gamma_{1}[(1-$ $\left.\left.\boldsymbol{\nu}_{1}^{2}\right) \check{\mathbf{i}}-\boldsymbol{\nu}_{1} \boldsymbol{\nu}_{2} \check{\mathbf{j}}\right]$, where $\check{\mathbf{i}}$ and $\check{\mathbf{j}}$ are unitary vectors along the $x_{1}$ and $x_{2}$ directions respectively.

A spherical droplet of radius $R$ is placed at the center of the domain. Since inertia is neglected the droplet instantaneously exhibits a rigid translation to regions of lower $\gamma$ at constant speed given by

$$
U=\frac{2}{15} \frac{\gamma_{1} R}{\mu}
$$




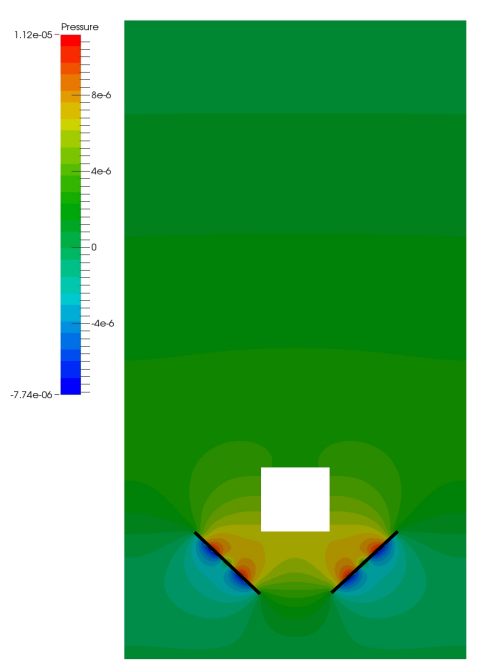

$t=1 \mathrm{~s}$

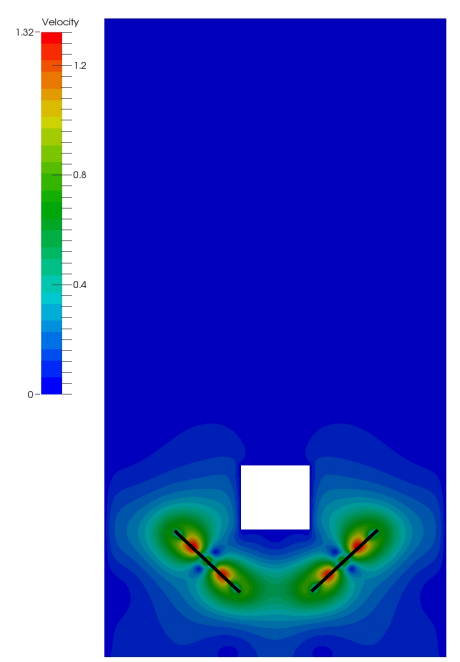

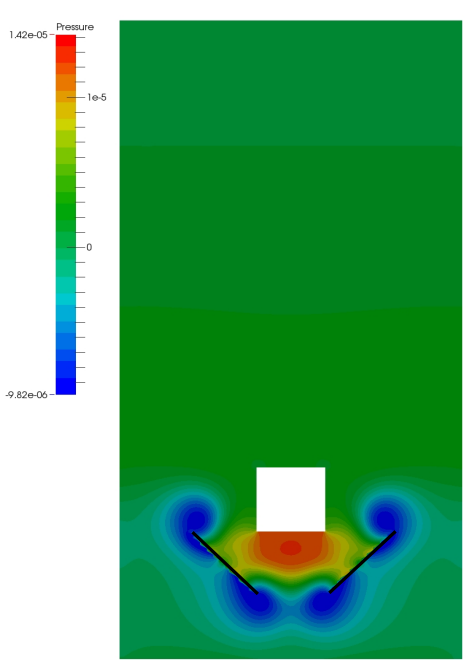

$t=3 \mathrm{~s}$

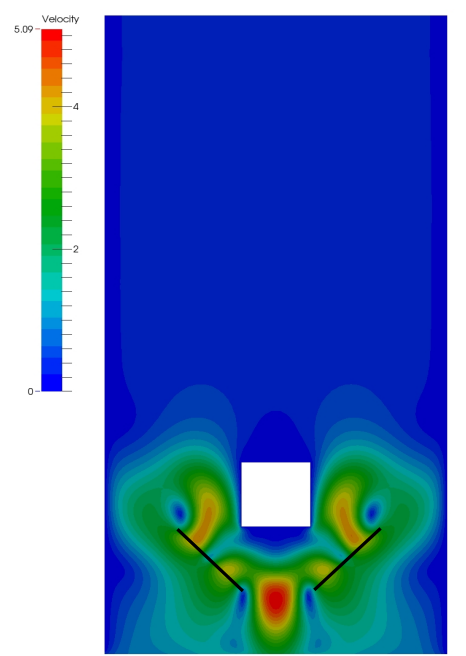

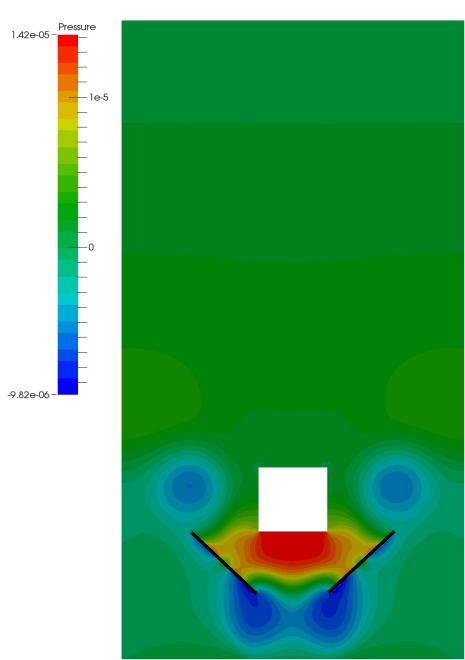

$t=5 \mathrm{~s}$

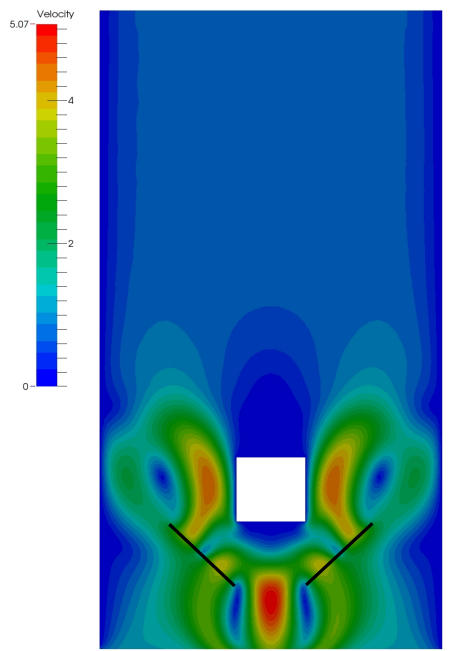

Figure 12. Pressure and velocity field at different times for the actuator disks inducing transient flow around a square obstacle by considering inertial effects.

Figure 17 shows the problem setting and relevant definitions. An analytical solution for this problem can be found in [23] and its numerical resolution by the finite element method was tackled in [14]. Setting $\mathbf{v}=\mathbf{u}-U \check{\mathbf{i}}$ the velocity and the pressure fields in each region read

$$
\left\{\begin{array}{l}
v_{r}^{+}=-U \cos \theta\left(1-\frac{R^{3}}{r^{3}}\right) \\
v_{\theta}^{+}=U \sin \theta\left(1+\frac{R^{3}}{2 r^{3}}\right) \quad \text { if } \quad r>R \\
p^{+}=p_{\infty}
\end{array}\right.
$$



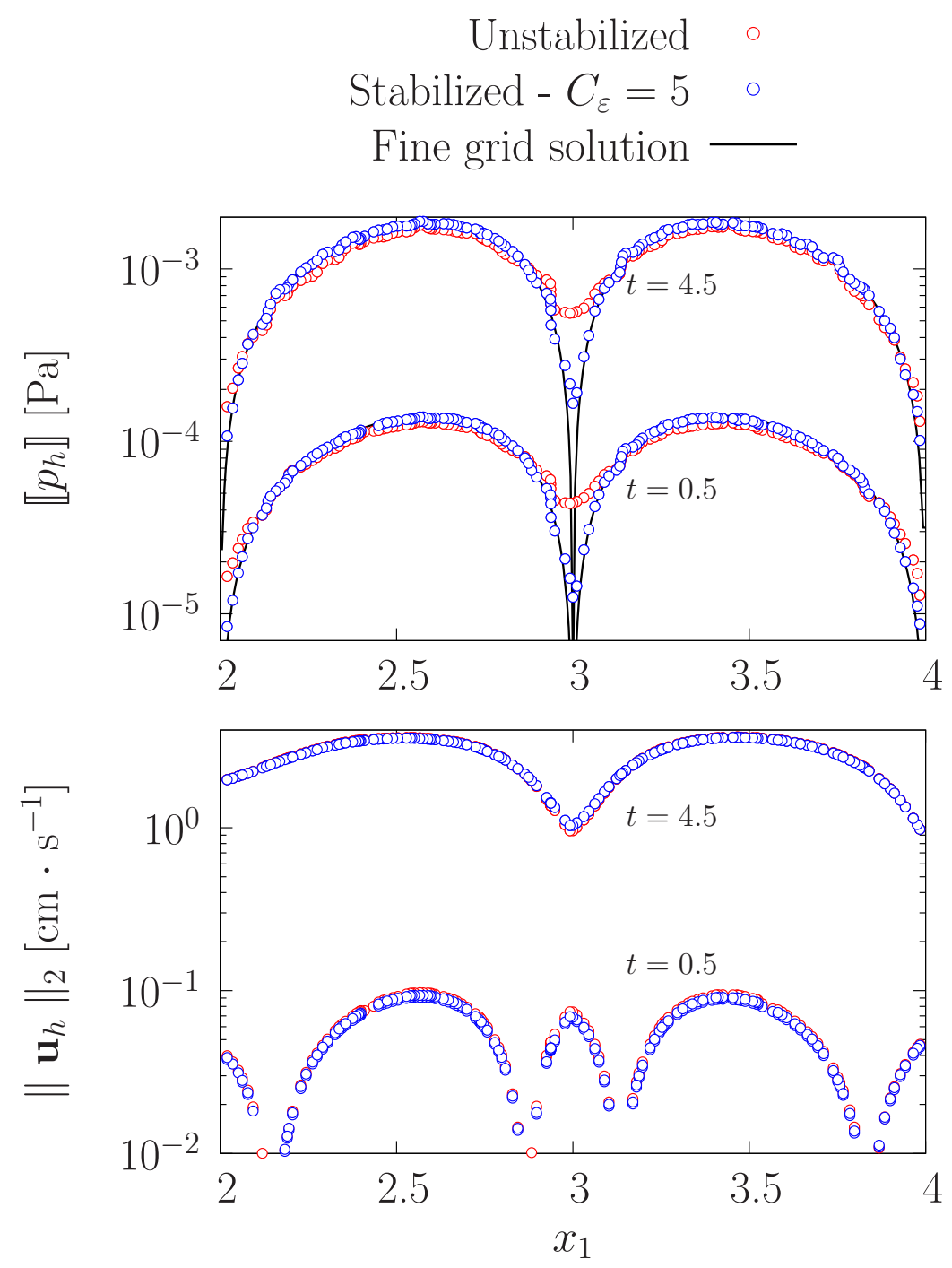

Figure 13. Pressure jump compared to the applied force (top) and magnitude of velocity field $\left\|\mathbf{u}_{h}\right\|_{2}$ (bottom) along the left interface at two different times for the actuator disks inducing transient flow around a square obstacle by considering inertial effects.

$$
\left\{\begin{array}{l}
v_{r}^{-}=\frac{3}{2} U \cos \theta\left(1-\frac{r^{2}}{R^{2}}\right) \\
v_{\theta}^{-}=3 U \sin \theta\left(\frac{r^{2}}{R^{2}}-\frac{1}{2}\right) \quad \text { if } \quad r<R \\
p^{-}=p_{\infty}+\frac{2}{R}\left(\gamma_{0}-\gamma_{1} r \cos \theta\right)
\end{array}\right.
$$

where the superscript + refers to the region outside the droplet and the superscript - refers to the region inside it and $(r, \theta)$ are the polar coordinates (see Figure 17). The velocity is continuous along $\Sigma_{h}$, and its radial component for $r=R$ is identically zero, thus preserving the droplet's shape. The pressure in turn exhibits a jump across $\Sigma$ depending linearly on $x_{1}$ 


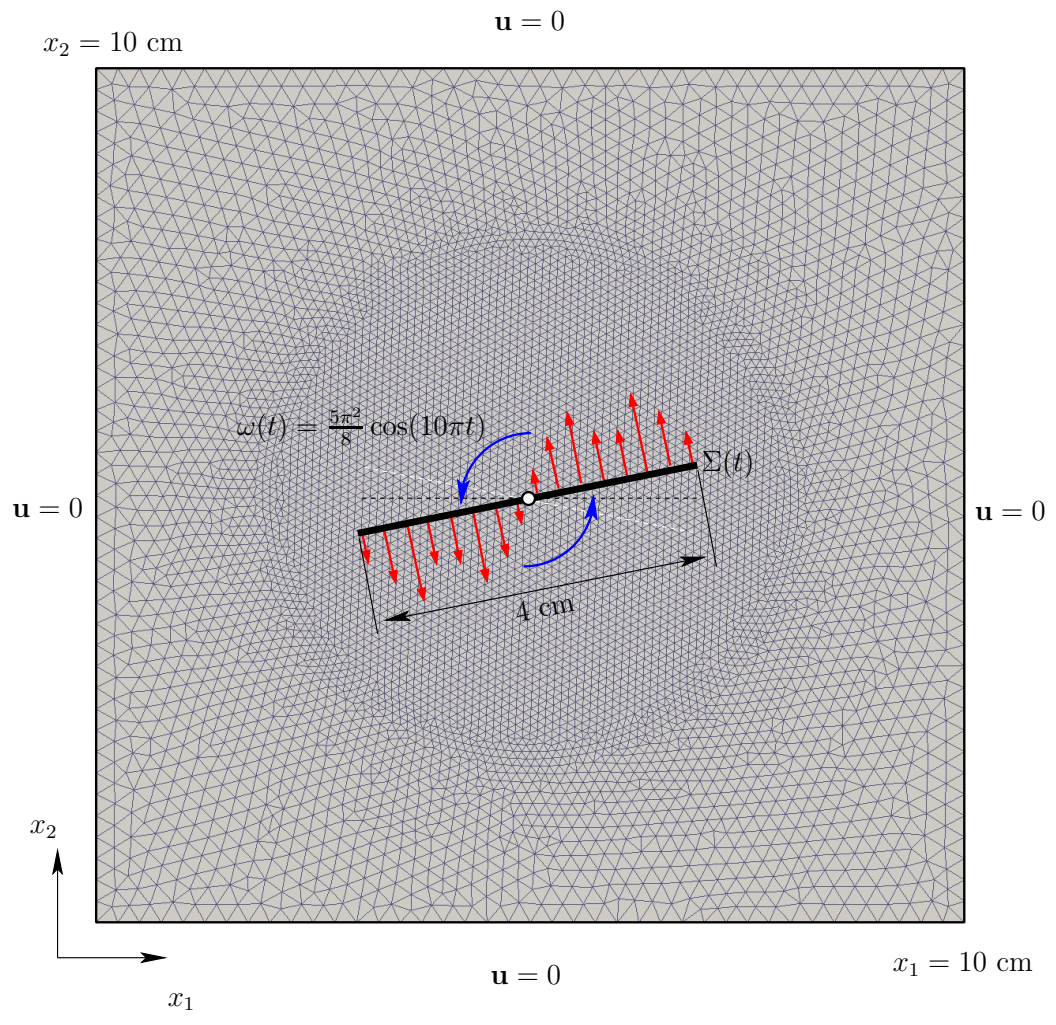

Figure 14. Problem setting for the rotating actuator disk.

$$
\llbracket p \rrbracket=\frac{2}{R}\left(\gamma_{0}-\gamma_{1} x_{1}\right)
$$

The singular force on $\Sigma_{h}$ is directly enforced considering an uniform mean curvature $\kappa=2 / R$ along the interface. This is to avoid any numerical error related to the computation of $\kappa$ that may pollute the results. The momentum equation in variational form thus reads

$$
\mu\left(\nabla \mathbf{u}_{h}, \nabla \mathbf{v}\right)_{\Omega_{h}}-\left(p_{h}, \operatorname{div} \mathbf{v}\right)_{\Omega_{h}}=(-\gamma \kappa, \mathbf{v} \cdot \boldsymbol{\nu})_{\Sigma}+\left(\nabla_{S} \gamma, \mathbf{v}\right)_{\Sigma}
$$

This approach is different from the one used in [14] in which the identity

$$
\int_{\Sigma}\left(-\gamma \kappa \boldsymbol{\nu}+\nabla_{S} \gamma\right) \cdot \mathbf{v} d \Sigma=-\int_{\Sigma} \gamma \nabla_{S} \cdot \mathbf{v} d \Sigma=-\int_{\Sigma} \gamma \mathbf{P}: \nabla \mathbf{v} d \Sigma
$$

is used (see [14] for more details). We solve this problem with an axisymmetric code. The computational domain is the rectangular region $\Omega=[0, L] \times[0, H]$, where $L=12 R$ and $H=4 R$. We consider $R=0.25$, $\mu=1, \gamma_{0}=1.5, \gamma_{1}=1$ and $p_{\infty}=0$. The symmetry axis is considered to be the line $x_{2}=0$. On the rest of $\partial \Omega_{h}$, we prescribe the exact solution (28)-(29) as Dirichlet conditions on $\mathbf{u}_{h}$ and $p_{h}$ when assembling the discrete linear system. The computational domain is initially discretized with 6000 triangles to which we assign a mesh size $h=0.03334$. From this, a sequence of meshes is built by uniform refinement. We display in Figure 18 streamlines for the velocity $\mathbf{v}\left(=\mathbf{u}_{h}-U \check{\mathbf{1}}\right)$ and contours of velocity and pressure. Figure 19 shows the pressure jump along the interface using the proposed method. for the second mesh of the sequence and the surface tension force $\varphi$ imposed. Figure 20 shows the $L^{2}\left(\Omega_{h}\right)$ error norm of pressure and the $H^{1}\left(\Omega_{h}\right)$ error norm of velocity. The error in the pressure field behaves clearly better in the stabilized case. The observed convergence rate is nearly an $O\left(h^{1.5}\right)$. As for the velocity error, the 


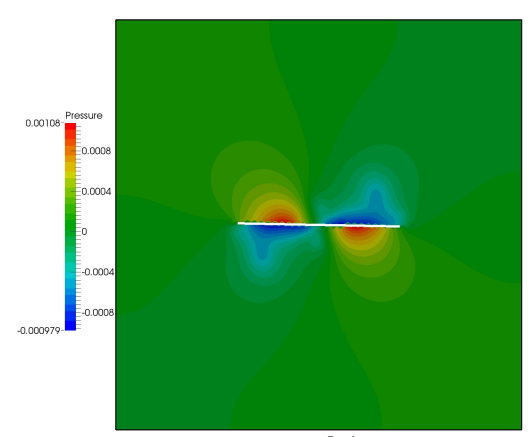

$t=0.1 \mathrm{~s}$
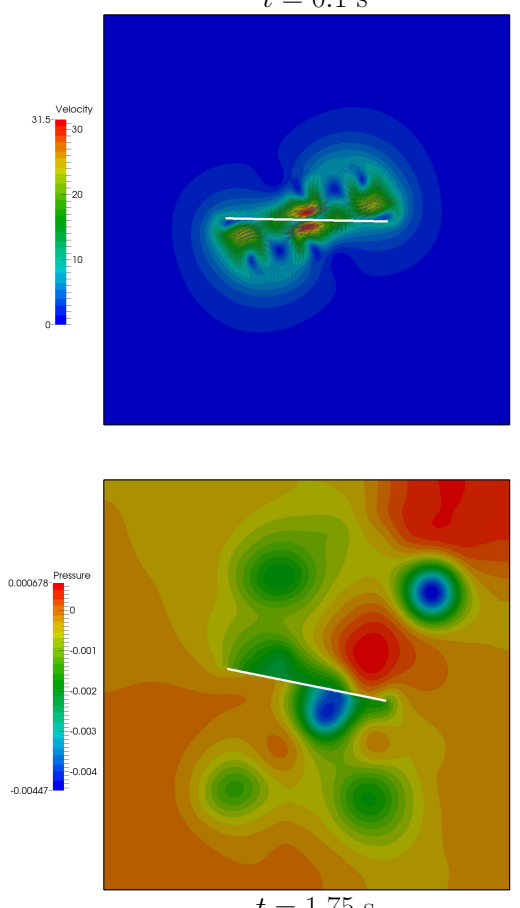

$t=1.75 \mathrm{~s}$

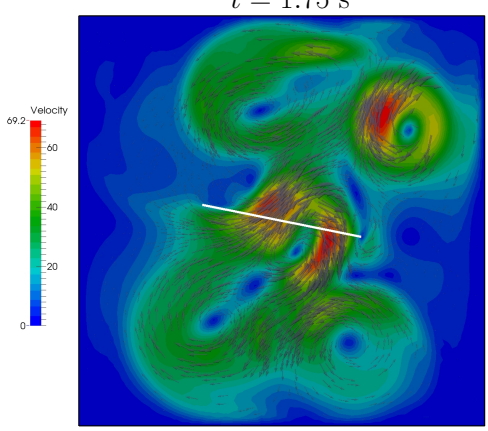

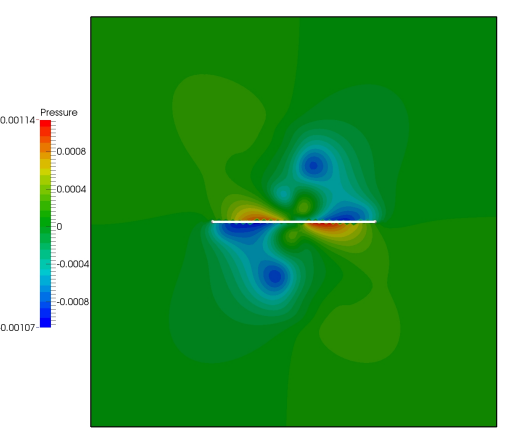

$t=0.5 \mathrm{~s}$
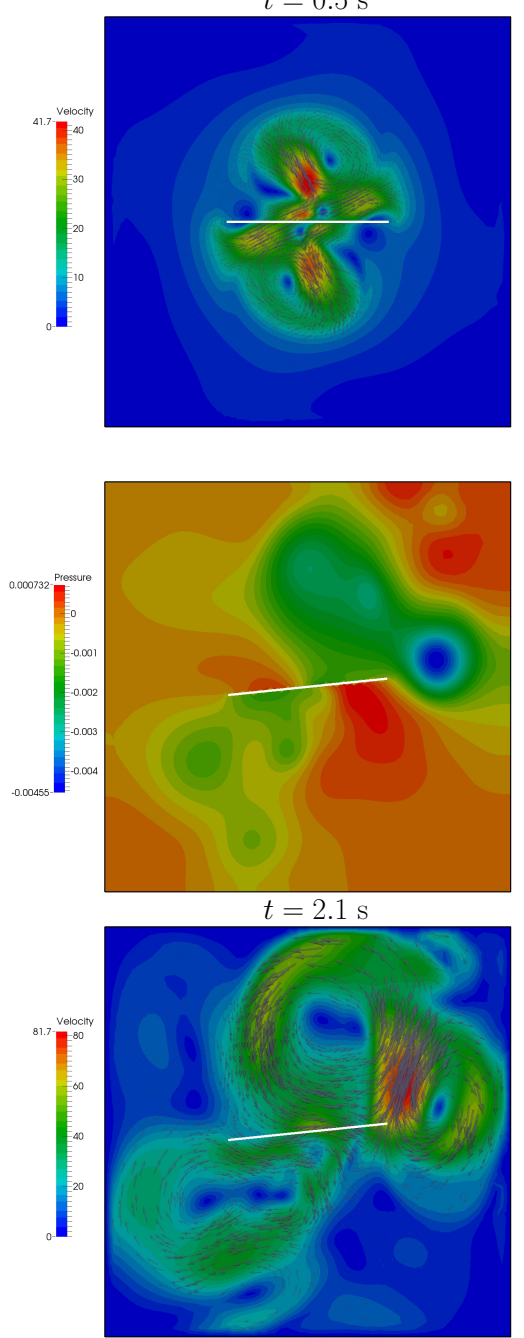

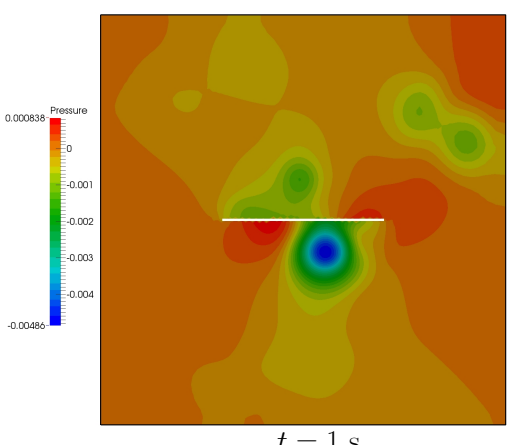

$t=1 \mathrm{~s}$
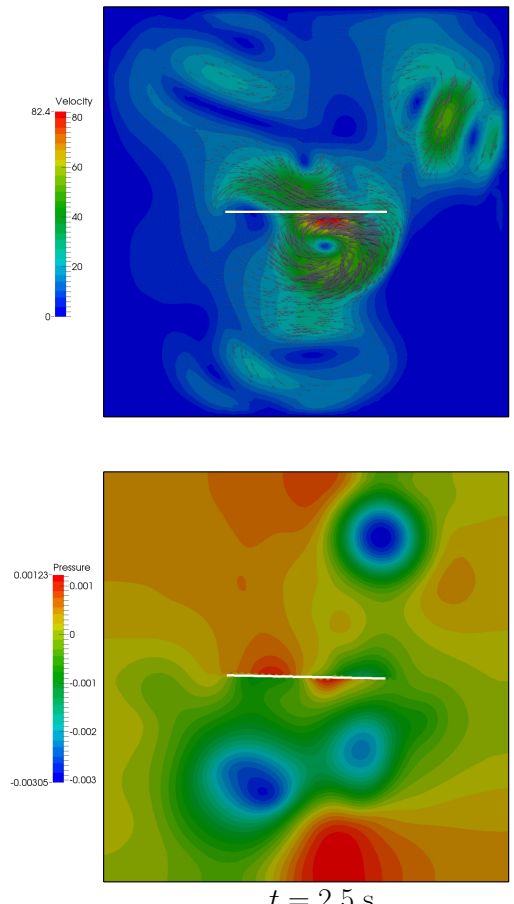

$t=2.5 \mathrm{~s}$

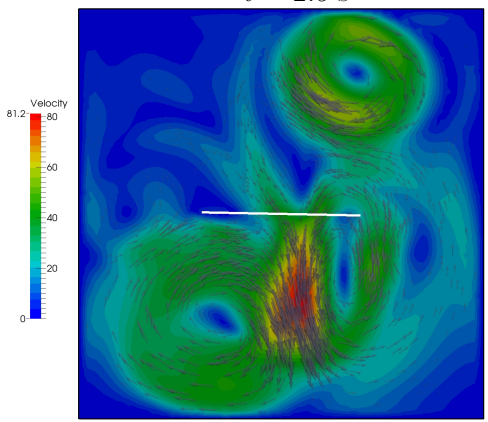

Figure 15. Pressure and velocity field at different times for the rotating disk problem taking $a=2 \times 10^{-3}$ Pa for amplitude of the force in Eq. (23). 


\section{Unstabilized -}

Stabilized $-C_{\varepsilon}=3$
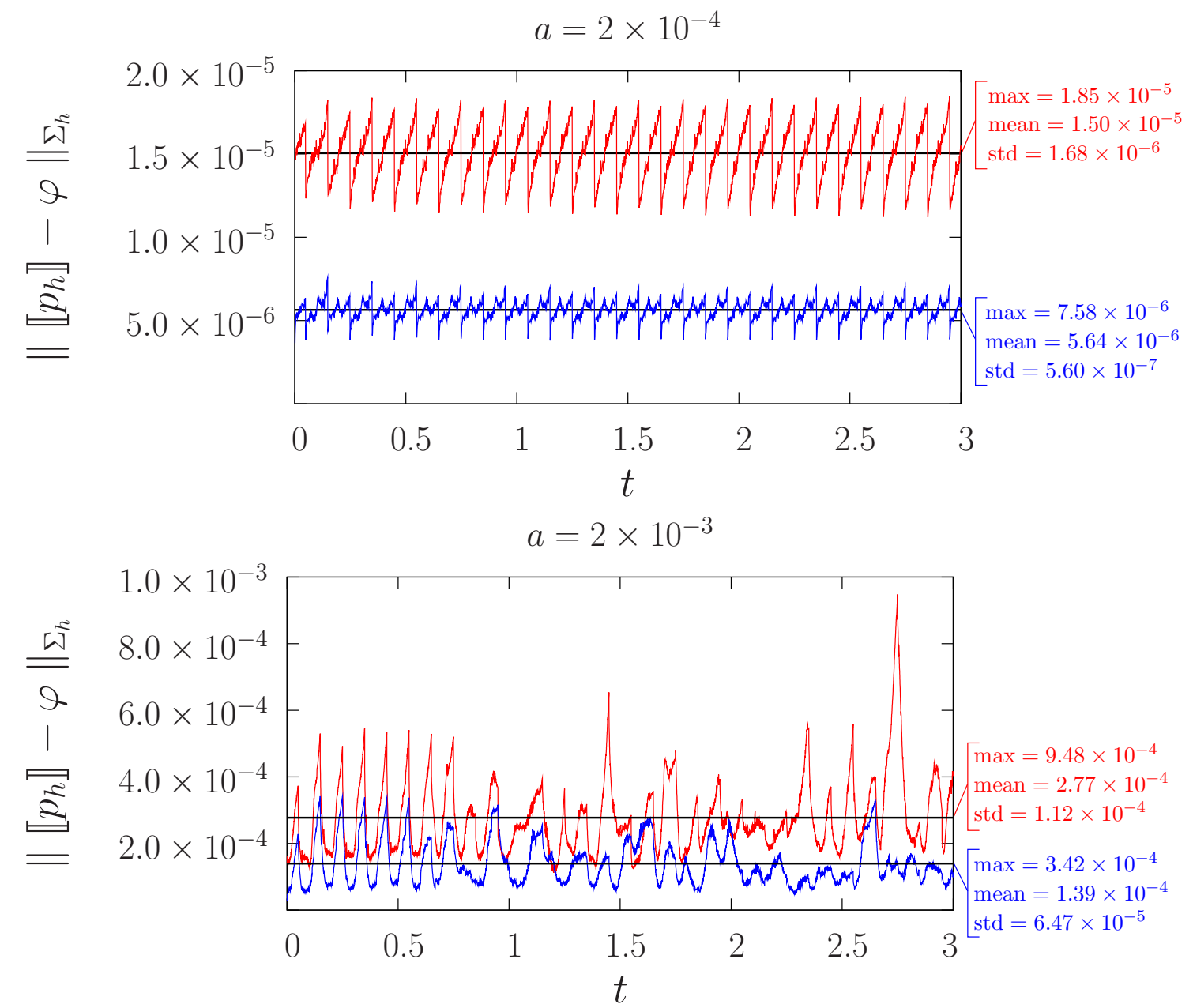

Figure 16. Error in the pressure error jump along $\Sigma$ as a function of time for the rotating actuator disk problem.

results are almost identical. The suboptimal order observed in this case $\left(\sim h^{0.5}\right)$ is a consequence of the discontinuity in the velocity gradient (cf. Figure 18) as a result of a non-zero tangential singular force on $\Sigma$. The discrete space for velocity is simply made up of standard piecewise linear continuous functions, with no special treatment in elements cut by the interface, as in the case of the pressure, for which the space proposed in [11] is being employed. As already mentioned, a possibility to deal with such kinks is the use of enrichment functions near $\Sigma_{h}$ or the XFEM method (see e.g. [27]). Statically condensable enrichments to tackle problems with weak discontinuities have also been proposed recently in [29], although, the authors only considered examples involving scalar unknowns. 


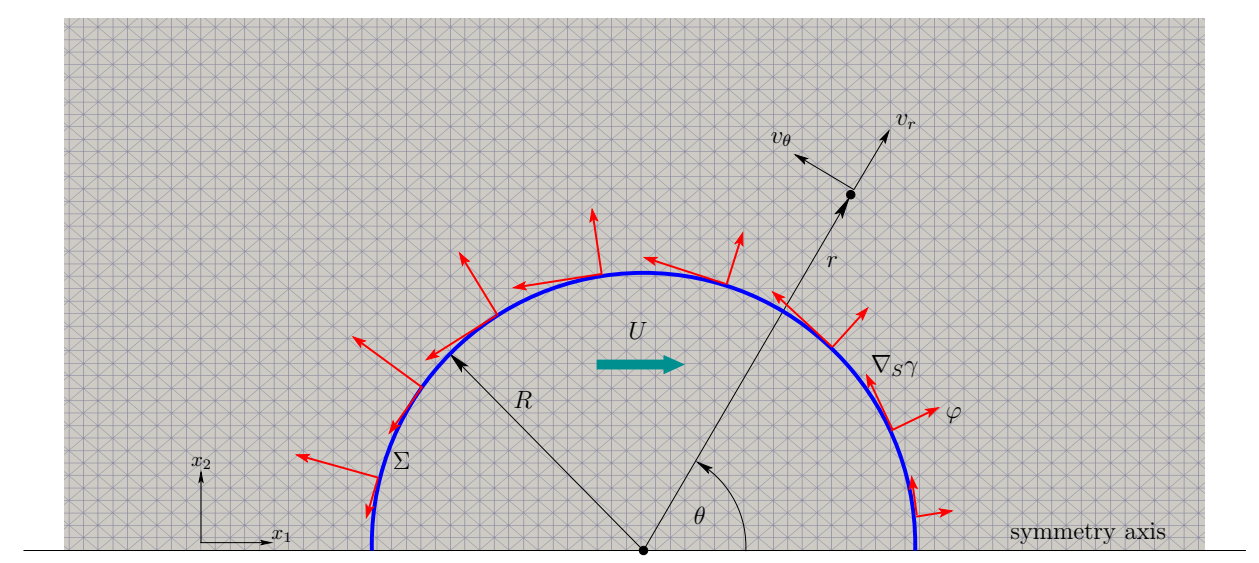

$\left(x_{1}=0, x_{2}=0\right)$

Figure 17. Problem setting for the thermocapillary (Marangoni) migration of a droplet.

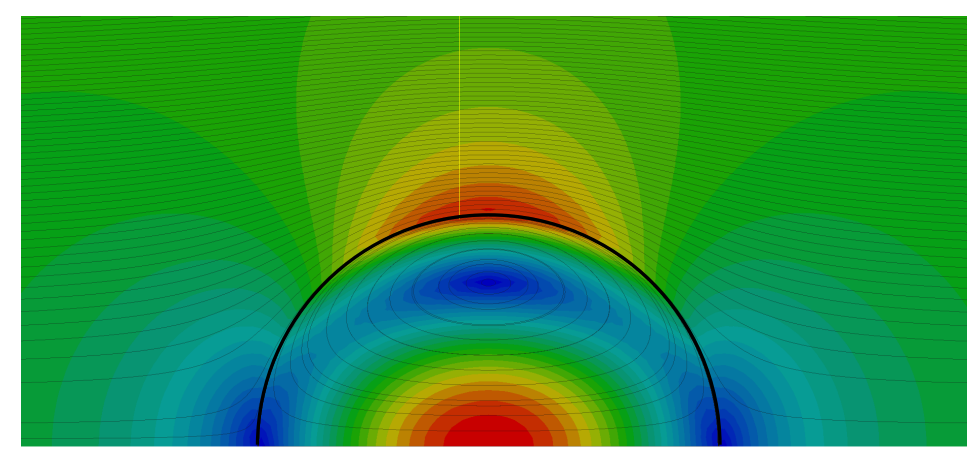

$\mathbf{v}=\mathbf{u}-\mathbf{U} \mathbf{i}$
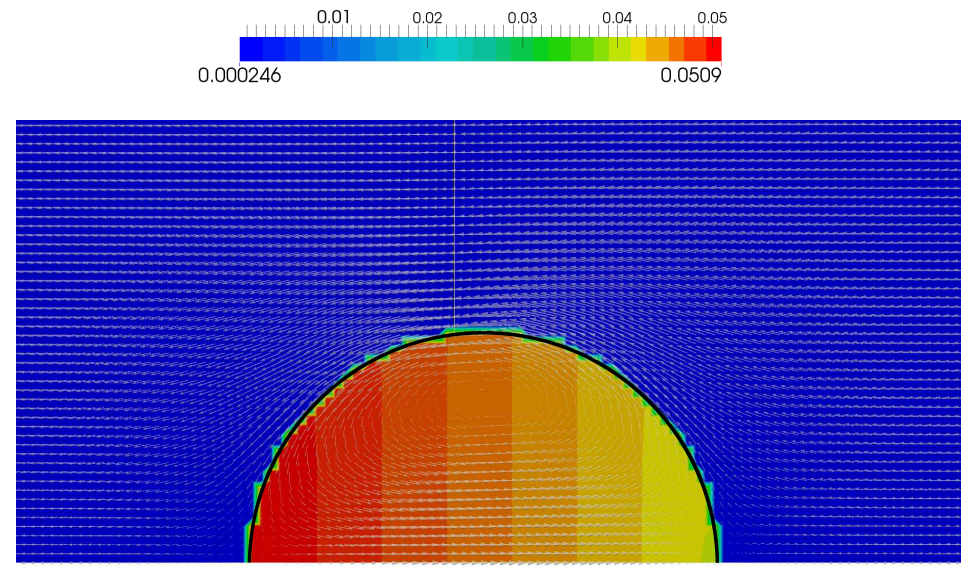

pressure

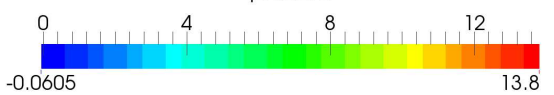

Figure 18. Contours of velocity $\mathbf{v}=\mathbf{u}_{h}-U \check{\mathbf{i}}$ (top) and pressure $p$ (bottom) for the thermocapillary migration of a droplet. 


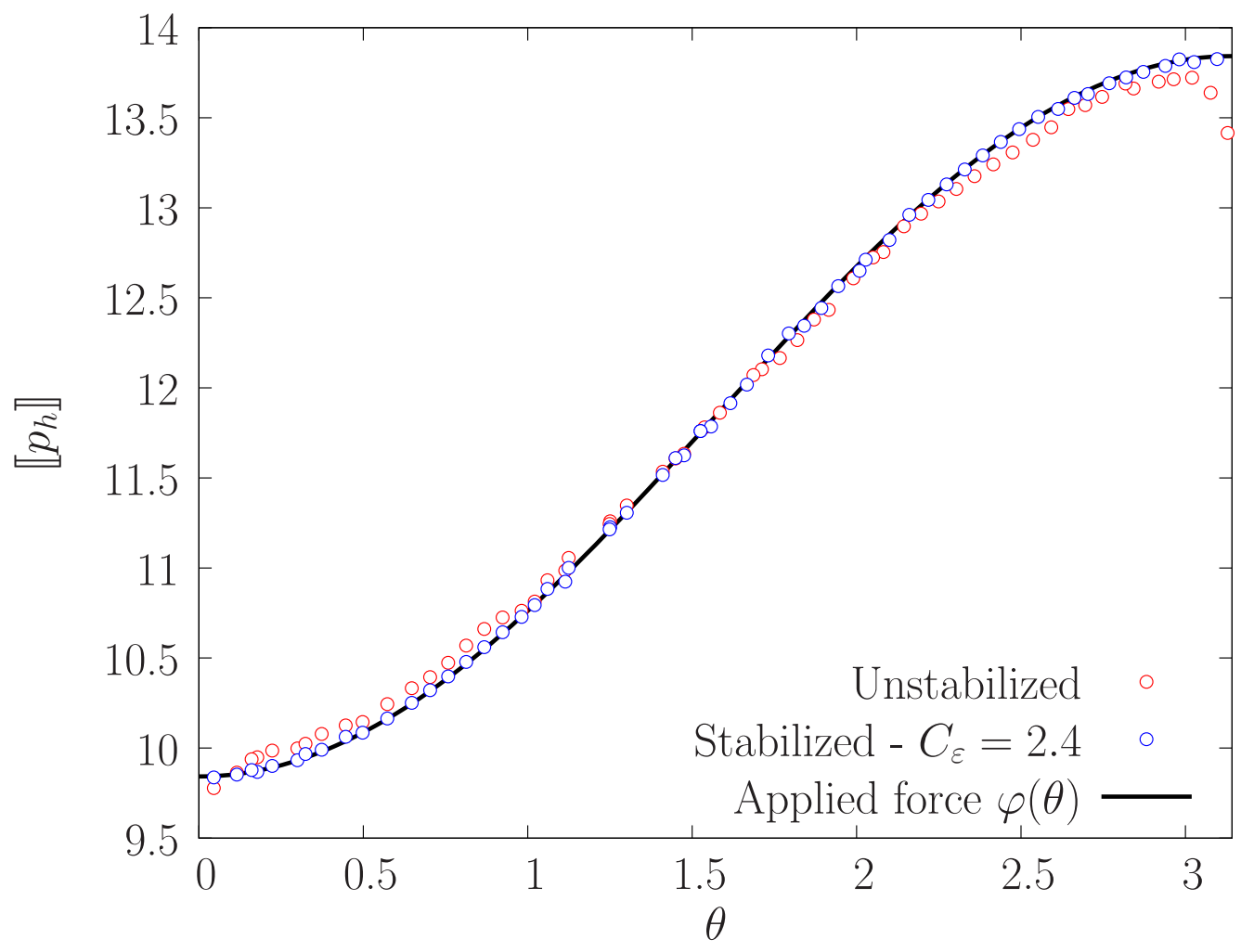

Figure 19. Singular force and pressure jump along $\Sigma$ for the thermocapillary migration of a droplet for the the second mesh of the sequence.

\section{Conclusions}

In this work we have proposed and extensively tested a finite element formulation for viscous incompressible flows in two dimensions, designed to simulate pressure embedded discontinuities along interfaces, owing, for instance, to actuator disks and surface tension effects. The new finite element method is a combination of the ASGS proposed by Codina [22] combined with the pressure jump stabilization technique studied in [12] and [13] (there, from the strict theoretical point of view) as applied to the Stokes system. The pressure jump stabilization is easy to implement in any standard finite element code, since it is based on the standard $P_{1}$ - i.e. continuous piecewise linear - interpolation of both velocity and pressure, with a local modification of the pressure representation proposed in [11], allowing for discontinuities along interfaces arbitrarily located with respect to the mesh. This formulation contains the basic ingredients considered in $[12,13]$, that is, a stabilization depending on a strictly positive parameter $\delta$, in order to circumvent the Babuška-Brezzi condition violated by the $P_{1}-P_{1}$ combination, but is much more general and allows to study more challenging problems such as those governed by the Navier-Stokes equations, including inertial and transient effects The pressure jump stabilization term depends on a positive real parameter $\varepsilon$ which is basically proportional to the mesh step size in order to reinforce the relation stating that the pressure jump across the interface is either a prescribed force or a part of the solution process itself.

It is noteworthy that in all cases that we have studied, the observed convergence rate of the pressure in the $L^{2}$-norm is significantly greater than the theoretical $O(h), h$ being the maximum mesh step size. 

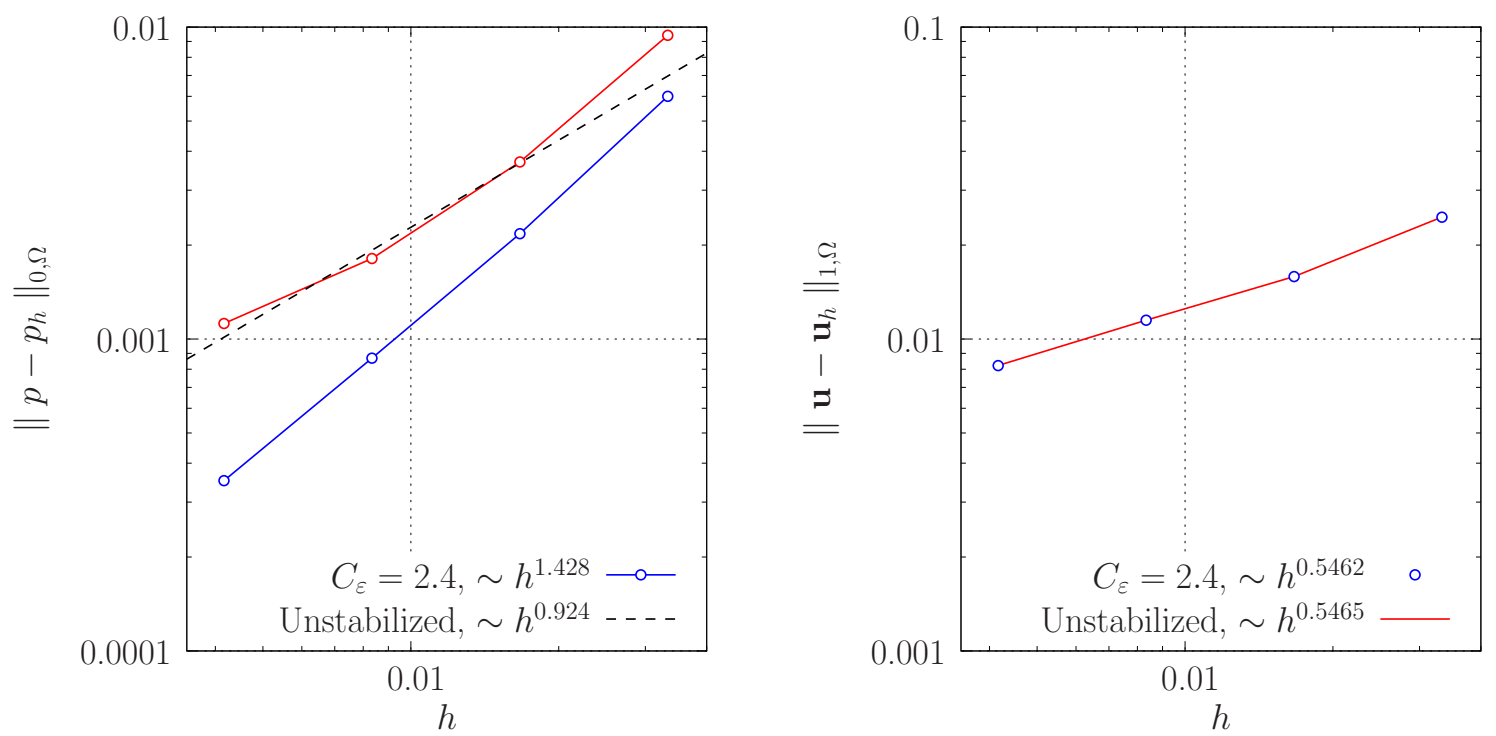

Figure 20. $L^{2}\left(\Omega_{h}\right)$ error of pressure (left) and $H^{1}\left(\Omega_{h}\right)$ error of velocity (right) for the thermocapillary migration of a droplet for the unstabilized method (red) and stabilized method (blue). The estimated convergence rate in each case is also indicated.

This rate even attained in some cases the theoretical $O\left(h^{1.5}\right)$ rate of the interpolation error using our discontinuous pressure space. The main focus of the numerical experiments has been the simulation of actuator disks. First, the convergence properties of the scheme have been assessed in an academic example and later on, considering a more realistic situation, the accuracy and robusteness of the method has been demonstrated in problems involving non-negligible inertial and transient effects. The method was also tested on a totally different field, by considering a problem of interest in microfluidics involving the thermocapillary Marangoni migration of a droplet and obtained an improved convergence rate for pressure with respect to the unstabilized case and no order erosion for velocity. The main conclusion of our numerical study is that the proposed method is always more effective in the computation of a pressure field accurately fulfilling the jump condition. By using the proposed formulation, more accurate numerical solutions can be expected without any substantial increase of computational effort and any deterioration of the velocity field.

\section{Acknowledgements}

The authors gratefully acknowledge the financial support received from both FAPESP (grants 2014/192491, 2013/07375-0 - Cepid-CeMEAI) and CNPq (grants 307996/2008-5, 447607/2014-6 and 308728/2013-0).

\section{References}

[1] P.-E. Réthoré, P. van der Laan, N. Troldborg, F. Zahle, N. Sørensen, Verification and validation of an actuator disc model, Wind Energy 17 (2014) 919-937.

[2] B. W.M.J., M. Harrison, A. Bahaj, Accuracy of the actuator disc-RANS approach for predicting the performance and wake of tidal turbines, Trans R Soc A 371 (20120293). 
[3] I. Ammara, C. Leclerc, C. Masson, A Viscous Three-Dimensional Differential/Actuator-Disk Method for the Aerodynamic Analysis of Wind Farms, Journal of Solar Energy Engineering 124 (2002) 345-356.

[4] E. Burman, P. Hansbo, Fictitious domain finite element methods using cut elements: II. A stabilized Nitsche method, Appl. Numer. Math. 62 (2012) 328-341.

[5] E. Burman, P. Hansbo, Fictitious domain methods using cut elements: III. A stabilized Nitsche method for stokes' problem, ESAIM: Math. Model. Numer. Anal. 48 (2014) 859-874.

[6] P. Hansbo, M. G. Larson, S. Zahedi, A cut finite element method for a stokes interface problem, Applied Numerical Mathematics 85 (2014) 90-114.

[7] S. Gross, A. Reusken, An extended pressure finite element space for two-phase incompressible flows with surface tension, J. Comput. Phys. 224 (2007) 40-58.

[8] A. Reusken, Analysis of an extended pressure finite element space for two-phase incompressible flows, Comput. Visual. Sci. 11 (2008) 293-305.

[9] K. Kamran, R. Rossi, E. Oñate, A locally extended finite element method for the simulation of multi-fluid flows using the particle level set method, Computer Methods in Applied Mechanics and Engineering 294 (2015) 1-18.

[10] R.A. Adams. Sobolev Spaces, Academic Press, New York, 1975.

[11] R. Ausas, F. Souza and G. Buscaglia. An improved finite element space for discontinuous pressures, Computer Methods in Applied Mechanics and Engineering 199 (2010), 1019-1031.

[12] G. Buscaglia and V. Ruas, Finite element methods for the Stokes system with interface pressure discontinuities, IMA Journal of Numerical Analysis, 35 (2015), 220-238.

[13] G. Buscaglia and V. Ruas, Approximation of the multi-dimensional Stokes system with embedded pressure discontinuities. Advances in Computational Mathematics, 41 (2014), 599-634.

[14] G. Buscaglia and R. F. Ausas. Variational formulations for surface tension, capillarity and wetting, Computer Methods in Applied Mechanics and Engineering 200 (2011), 3011-3025.

[15] E. Cartan, Leçons sur la géométrie des espaces de Riemann, Gauthier-Villars, Paris, 1928.

[16] P.G.Ciarlet, The Finite Element Method for Elliptic Problems, North Holland, Amsterdam, 1978.

[17] J. Douglas and J. Wang. An Absolutely Stabilized Finite Element Method for the Stokes Problem, Mathematics of Computation, 52-186 (1989), 495-508.

[18] L. Franca, T.J.R. Hughes and R. Stenberg. Stabilized Finite Element Methods, in: Incompressible Computational Fluid Dynamics, M.D. Gunzburger and R.A. Nicolaides eds., Cambridge University Press, p. 87-107, 1994.

[19] V. Girault and P.A. Raviart. Finite Element Methods for Navier-Stokes Equations, Springer, N.Y., 1986.

[20] T.J.R. Hughes, L. Franca and M. Balestra. A new finite element formulation for computational fluid dynamics: V. Circumventing the Babuška-Brezzi condition: A stable Petrov-Galerkin formulation of the Stokes problem accommodating equal-order interpolations, Comp. Meths. Appl. Mechs. Engin., 59 (1986), 85-99.

[21] C. Pommerenke. Boundary Behaviour of Conformal Maps, Springer Verlag, Berlin, 1992.

[22] R. Codina. A stabilized finite element method for generalized stationary incompressible flows, Computer Methods in Applied Mechanics and Engineering 190 (2001), 2681-2706.

[23] R. Balasubramaniam, An-Ti Chai, Thermocapillary migration of droplets: an exact solution for small Marangoni numbers, J. Colloid Interf. Sci., 119, 1987.

[24] R. Ausas, G. Buscaglia and S. Idelsohn. A new enrichment space for discontinuous pressures, Int. J. Num. Meth. Fluids 70(7) (2012), 829-850.

[25] R. Mikkelsen, Actuator disc methods applied to wind turbines, Ph.D. thesis, Technical University of Denmark, 2003.

[26] PD. Minev, T. Chen and K. Nandakumar. A finite element technique for multifluid incompressible flow using Eulerian grids. J. Comput. Phys. 2003; 187:255-273.

[27] T. Belytschko, N. Moës, S. Usui, C. Parimi. Arbitrary discontinuities in finite elements. Int. J. Numer. Meth. Engng $2001 ; \mathbf{5 0 : 9 9 3 - 1 0 1 3 . ~}$

[28] AH. Coppola-Owen and R. Codina. Improving eulerian two-phase flow finite element approximation with discontinuous gradient pressure shape functions. Int. J. Numer. Meth. Fluids 2005; 49:1287-1304.

[29] S. R. Idelsohn, J. M. Gimenez, J. Marti, N. M. Nigro, Elemental enriched spaces for the treatment of weak and strong discontinuous fields, Computer Methods in Applied Mechanics and Engineering 313, 535 - 559, 2017. 